\title{
Symmetric implication zroupoids and identities of Bol-Moufang type
}

\author{
Juan M. Cornejo and Hanamantagouda P. Sankappanavar
}

This paper has been pubished by Soft Comput onlline on 25 October 2017. DOI 10.1007/s00500-017-2869-Z

\begin{abstract}
An algebra $\mathbf{A}=\langle A, \rightarrow, 0\rangle$, where $\rightarrow$ is binary and 0 is a constant, is called an implication zroupoid ( $\mathcal{I}$-zroupoid, for short) if $\mathbf{A}$ satisfies the identities: (I): $(x \rightarrow y) \rightarrow z \approx\left(\left(z^{\prime} \rightarrow x\right) \rightarrow(y \rightarrow z)^{\prime}\right)^{\prime}$, and $\left(\mathrm{I}_{0}\right)$ : $0^{\prime \prime} \approx 0$, where $x^{\prime}:=x \rightarrow 0$. An implication zroupoid is symmetric if it satisfies the identities: $x^{\prime \prime} \approx x$ and $\left(x \rightarrow y^{\prime}\right)^{\prime} \approx\left(y \rightarrow x^{\prime}\right)^{\prime}$. An identity is of Bol-Moufang type if it contains only one binary operation symbol, one of its three variables occurs twice on each side, each of the other two variables occurs once on each side, and the variables occur in the same (alphabetical) order on both sides of the identity.

In this paper we make a systematic analysis of all 60 identities of Bol-Moufang type in the variety $\mathcal{S}$ of symmetric $\mathcal{I}$-zroupoids. We show that 47 of the subvarieties of $\mathcal{S}$, defined by the identities of BolMoufang type are equal to the variety $\mathcal{S} \mathcal{L}$ of $\vee$-semilattices with the least element 0 and, one of them is equal to $\mathcal{S}$. Of the remaining 12, there are only 3 distinct ones. We also give an explicit description of the poset of the (distinct) subvarieties of $\mathcal{S}$ of Bol-Moufang type.
\end{abstract}

\section{Introduction}

In 1934, Bernstein gave a system of axioms for Boolean algebras in Be34] using implication alone. Even though his system was not equational, it is not hard to see that one could easily convert it into an equational one by using an additional constant. In 2012, the second author extended this "modified Bernstein's theorem" to De Morgan algebras in San12. Indeed, he shows in San12 that the varieties of De Morgan algebras, Kleene algebras, and Boolean algebras are term-equivalent, respectively, to the varieties, $\mathcal{D} \mathcal{M}$, $\mathcal{K} \mathcal{L}$, and $\mathcal{B A}$ (defined below) whose defining axioms use only an implication 
$\rightarrow$ and a constant 0 . The primary role played by the identity (I): $(x \rightarrow$ $y) \rightarrow z \approx\left[\left(z^{\prime} \rightarrow x\right) \rightarrow(y \rightarrow z)^{\prime}\right]^{\prime}$, where $x^{\prime}:=x \rightarrow 0$, in the axiomatization of each of those new varieties motivated the second author to introduce a new equational class of algebras called "Implication zroupoids" in San12. It also turns out that this new variety contains the variety $\mathcal{S} \mathcal{L}$ (defined below) which is shown (see CS16]) to be term-equivalent to the variety of $\checkmark$-semilattices with the least element 0 .

DEFINITION 1.1. An algebra $\mathbf{A}=\langle A, \rightarrow, 0\rangle$, where $\rightarrow$ is binary and 0 is a constant, is called a zroupoid. A zroupoid $\mathbf{A}=\langle A, \rightarrow, 0\rangle$ is an Implication zroupoid ( $\mathcal{I}$-zroupoid, for short) if $\mathbf{A}$ satisfies:

(I) $(x \rightarrow y) \rightarrow z \approx\left(\left(z^{\prime} \rightarrow x\right) \rightarrow(y \rightarrow z)^{\prime}\right)^{\prime}$, where $x^{\prime}:=x \rightarrow 0$,

$\left(\mathrm{I}_{0}\right) 0^{\prime \prime} \approx 0$.

$\mathcal{I}$ denotes the variety of implication zroupoids. The varieties $\mathcal{D} \mathcal{M}, \mathcal{I}_{1,0}, \mathcal{C}$ and $\mathcal{S} \mathcal{L}$ are defined relative to $\mathcal{I}$, respectively, by the following identities:

$$
\begin{array}{ll}
(\mathrm{DM}) & (x \rightarrow y) \rightarrow x \approx x \text { (De Morgan Algebras); } \\
\left(\mathrm{I}_{1,0}\right) & x^{\prime} \approx x \\
(\mathrm{C}) & x \rightarrow y \approx y \rightarrow x \\
(\mathrm{SL}) & x^{\prime} \approx x \text { and } x \rightarrow y \approx y \rightarrow x
\end{array}
$$

The varieties $\mathcal{K} \mathcal{L}$ and $\mathcal{B} \mathcal{A}$ are defined relative to $\mathcal{D} \mathcal{M}$, respectively, by the following identities:

(KL) $\quad(x \rightarrow x) \rightarrow(y \rightarrow y) \approx y \rightarrow y$ (Kleene algebras);

(BA) $x \rightarrow x \approx 0^{\prime}$ (Boolean algebras).

As proved in $[$ San12], the variety $\mathcal{I}$ generalizes the variety of De Morgan algebras and exhibits several interesting properties; for example, the identity $x^{\prime \prime \prime} \rightarrow y \approx x^{\prime} \rightarrow y$ holds in $\mathcal{I}$. Several new subvarieties of $\mathcal{I}$ are also introduced and investigated in San12]. The (still largely unexplored) lattice of subvarieties of $\mathcal{I}$ seems to be fairly complex. In fact, Problem 6 of [San12] calls for an investigation of the structure of the lattice of subvarieties of $\mathcal{I}$.

The papers [CS16], CS16a] and CS16b] have addressed further, but still partially, the above-mentioned problem by introducing new subvarieties of $\mathcal{I}$ and investigating relationships among them. The (currently known) size of the poset of subvarieties of $\mathcal{I}$ is at least 24 ; but it is still unknown whether the lattice of subvarieties is finite or infinite. Two of the subvarieties of $\mathcal{I}$ 
are: $\mathcal{I}_{2,0}$ and $\mathcal{M C}$ which are defined relative to $\mathcal{I}$, respectively, by the following identities, where $x \wedge y:=\left(x \rightarrow y^{\prime}\right)^{\prime}$ :

$$
\begin{gathered}
x^{\prime \prime} \approx x ; \\
x \wedge y \approx y \wedge x .
\end{gathered}
$$

For a somewhat more detailed summary of the results contained in the above-mentioned papers, we refer the reader to the introduction of [CS16c].

DEFINITION 1.2. Let $\mathbf{A} \in \mathcal{I}$. A is involutive if $A \in \mathcal{I}_{2,0}$. A is meetcommutative if $A \in \mathcal{M C}$. $\mathbf{A}$ is symmetric if $\mathbf{A}$ is both involutive and meetcommutative. Let $\mathcal{S}$ denote the variety of symmetric $\mathcal{I}$-zroupoids. In other words, $\mathcal{S}=\mathcal{I}_{2,0} \cap \mathcal{M C}$.

In the present paper we are interested in the subvarieties of $\mathcal{S}$ defined by certain weak associative laws, called "Bol-Moufang" laws. A precise definition of a weak associative law appears in [Ku96, which is essentially restated below.

DEFINITION 1.3. Let $n \in \mathbb{N}$ and let $\mathcal{L}:=\langle x\rangle$, where $\times$ is a binary operation symbol. A (groupoid) term in $\mathcal{L}$ is of length $n$ if the number of occurrences of variables (not necessarily distinct) is $n$. A weak associative law of length $n$ in $\mathcal{L}$ is an identity of the form $p \approx q$, where $p$ and $q$ are of length $n$ and contain the same variables that occur in the same order in both $p$ and $q$ (only the bracketing is possibly different).

We note that the identities of Bol-Moufang type investigated in [Fe69] are weak associative laws of length 4 in three distinct variables, with one of them repeated (see below for a more precise definition).

The following (general) problem presents itself naturally.

PROBLEM: Let $\mathcal{V}$ be a given variety of algebras (whose language includes a binary operation symbol, say, $\times$ ). Investigate the subvarieties of $\mathcal{V}$ defined by weak associative laws (with respect to $\times$ ) and their mutual relationships.

Special cases of the above problem have already been considered in the literature, wherein the weak associative laws are the identities of BolMoufang type, and the variety $\mathcal{V}$ is the variety of quasigroups or the variety of loops (see [Fe69], Ku96, PV05a, PV05b]).

[Fe69] has noted that there are 60 weak associative laws of length 4 in three distinct variables (with one variable repeated). Since the Moufang laws 
and the Bol identities were among those 60 laws, the author of that paper called such identities as those of Bol-Moufang type. For more information about these identities in the context of quasigroups and loops, see Ku96, PV05a, PV05b.

In this paper and in CS16d, we carry out a complete analysis of weak associative identities of lenth $\leq 4$, in the context of the variety $\mathcal{S}$ of symmetric implication zroupoids. We focus, in this paper, exclusively on the systematic study of the identities of Bol-Moufang type in the context of symmetric implication zroupoids, while [CS16d] will focus on the rest of the weak associative identities.

Without loss of generality, we will assume that the variables in $p$ and $q$ occur alphabetically. So, we can say (more explicitly) that an identity $p \approx q$, in the language $\langle\rightarrow\rangle$, is of Bol-Moufang type if:

(i) $p$ and $q$ contain the same three variables (chosen alphabetically),

(ii) the length of $p=4=$ the length of $q$,

(iii) the order in which the variables appear in $p$ is exactly the same as the order in which they appear in $q$.

Let $B$ denote the set of identities of Bol-Moufang type.

The systematic notation for the identities in $B$, presented below, was developed in PV05a].

Let $x, y, z$ be all the variables appearing in the identities of $B$. Without loss of generality, we can assume that they appear in the terms in the alphabetical order. Then, the 6 ways in which the 3 variables can form a word of length 4 are shown below:
A : $\quad$ xxyz;
B : $\quad x y x z$
C : xyyz;
D : xyzx;
E : xyzy;
F : xyzz.

It is clear that there are exactly 5 ways in which a word of size 4 can be bracketed, as given below:

1: $\quad o \rightarrow(o \rightarrow(o \rightarrow o))$;

2: $\quad o \rightarrow((o \rightarrow o) \rightarrow o)$;

3: $\quad(o \rightarrow o) \rightarrow(o \rightarrow o)$;

4: $\quad(o \rightarrow(o \rightarrow o)) \rightarrow o$; 
5: $\quad((o \rightarrow o) \rightarrow o) \rightarrow o$.

Let $\left(X_{i j}\right)$ with $X \in\{\mathrm{A}, \ldots, \mathrm{F}\}, 1 \leq i<j \leq 5$, denote the identity from $B$ whose left-hand side is bracketed according to $i$, and whose righthand side is bracketed according to $j$. For instance, $\left(A_{12}\right)$ is the identity $x \rightarrow(x \rightarrow(y \rightarrow z)) \approx x \rightarrow((x \rightarrow y) \rightarrow z)$. We note that $\mathrm{B}_{15}$ is a Moufang identity, while $\mathrm{E}_{25}$ is a Bol identity.

It is noted in Fe69] that there are $(6 \times(4+3+2+1)=) 60$ nontrivial identities in B.

We will denote by $\mathcal{X}_{i j}$ the subvariety of $\mathcal{S}$ defined by the identity $\left(X_{i j}\right)$.

We will show in Section 3 that 47 of these 60 varieties coincide with $\mathcal{S} \mathcal{L}$ (and hence with each other). In Section 4, we prove our main result that there are 4 nontrivial varieties of Bol-Moufang type that are distinct from each other. Furthermore, we describe explicitly the poset formed by them, together with the variety $\mathcal{B A}$ (which is contained in some of them).

We would like to acknowledge that the software "Prover 9/Mace 4" developed by McCune $[\mathrm{Mc}$ has been useful to us in some of our findings presented in this paper. We have used it to find examples and to check some conjectures.

\section{Preliminaries}

We refer the reader to the standard references [BD74, BS81] and [R74] for concepts and results used, but not explained, in this paper.

Recall from San12 that $\mathcal{S} \mathcal{L}$ is the variety of semilattices with a least element 0. It was shown in CS16 that $\mathcal{S} \mathcal{L}=\mathcal{C} \cap \mathcal{I}_{1,0}$.

The two-element algebras $\mathbf{2}_{\mathrm{s}}, \mathbf{2}_{\mathrm{b}}$ were introduced in San12. Their operations $\rightarrow$ are respectively as follows:

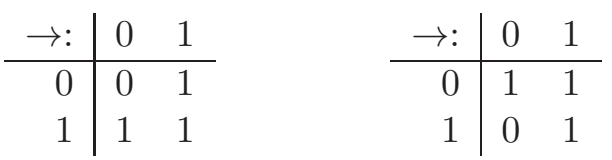

Notice that $\mathcal{V}\left(\mathbf{2}_{\mathbf{b}}\right)=\mathcal{B A}$. Recall also from [CS16, Corollary 11.4] that $\mathcal{V}\left(\mathbf{2}_{\mathbf{s}}\right)=\mathcal{S} \mathcal{L}$. The following lemma easily follows from the definition of $\wedge$ given earlier in the Introduction.

LEMMA 2.1. $\mathcal{M C} \cap \mathcal{I}_{1,0} \subseteq \mathcal{C} \cap \mathcal{I}_{1,0}=\mathcal{S} \mathcal{L}$.

LEMMA 2.2. [San12, Theorem 8.15] Let A be an $\mathcal{I}$-zroupoid. Then the following are equivalent: 

(a) $0^{\prime} \rightarrow x \approx x$,
(b) $x^{\prime \prime} \approx x$,
(c) $\left(x \rightarrow x^{\prime}\right)^{\prime} \approx x$,
(d) $x^{\prime} \rightarrow x \approx x$.

LEMMA 2.3. San12] Let $\mathbf{A} \in \mathcal{I}_{2,0}$. Then
(a) $x^{\prime} \rightarrow 0^{\prime} \approx 0 \rightarrow x$,
(b) $0 \rightarrow x^{\prime} \approx x \rightarrow 0^{\prime}$.

LEMMA 2.4. Let $\mathbf{A} \in \mathcal{I}_{2,0}$. Then $\mathbf{A}$ satisfies:
(a) $\left(x \rightarrow 0^{\prime}\right) \rightarrow y \approx\left(x \rightarrow y^{\prime}\right) \rightarrow y$,
(b) $x \rightarrow(0 \rightarrow x)^{\prime} \approx x^{\prime}$,
(c) $(y \rightarrow x) \rightarrow y \approx(0 \rightarrow x) \rightarrow y$,
(d) $(0 \rightarrow x) \rightarrow(0 \rightarrow y) \approx x \rightarrow(0 \rightarrow y)$,
(e) $x \rightarrow y \approx x \rightarrow(x \rightarrow y)$,
(f) $0 \rightarrow(0 \rightarrow x)^{\prime} \approx 0 \rightarrow x^{\prime}$,
(g) $0 \rightarrow\left(x^{\prime} \rightarrow y\right)^{\prime} \approx x \rightarrow\left(0 \rightarrow y^{\prime}\right)$,
(h) $0 \rightarrow(x \rightarrow y) \approx x \rightarrow(0 \rightarrow y)$,
(i) $0 \rightarrow\left(x \rightarrow y^{\prime}\right)^{\prime} \approx 0 \rightarrow\left(x^{\prime} \rightarrow y\right)$,
(j) $x \rightarrow\left(y \rightarrow x^{\prime}\right) \approx y \rightarrow x^{\prime}$
(k) $\left(x \rightarrow y^{\prime}\right)^{\prime} \rightarrow z \approx x \rightarrow(y \rightarrow z)$.

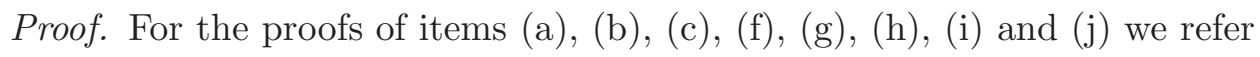
the reader to [CS16, Lemma 2.7]. The proofs of items (d) and (国) are given in [CS16a, Lemma 2.6], while the proof of the item (ㅌ) can be found in CS16c, Lemma 3.3].

LEMMA 2.5. Let $\mathbf{A} \in \mathcal{I}_{2,0}$ such that $\mathbf{A} \models 0 \rightarrow x \approx x$, then $\mathbf{A} \models(x \rightarrow$ $y)^{\prime} \approx x^{\prime} \rightarrow y^{\prime}$. 
Proof. Let $a, b \in A$. Hence, we have that

$$
\begin{aligned}
a^{\prime} \rightarrow b^{\prime} & =0 \rightarrow\left(a^{\prime} \rightarrow b^{\prime}\right) & & \text { by hyphotesis } \\
& =a^{\prime} \rightarrow\left(0 \rightarrow b^{\prime}\right) & & \text { by Lemma } 2.4(\underline{\mathrm{h}}) \\
& =0 \rightarrow\left(a^{\prime \prime} \rightarrow b\right)^{\prime} & & \text { by Lemma } 2.4(\mathrm{~g}) \\
& =0 \rightarrow(a \rightarrow b)^{\prime} & & \text { by }\left(\mathrm{I}_{2,0}\right. \\
& =(a \rightarrow b)^{\prime} & & \text { by hyphotesis. }
\end{aligned}
$$

This completes the proof.

\section{Symmetric $\mathcal{I}$-zroupoids of Bol-Moufang type}

Recall that the variety $\mathcal{S}=\mathcal{I}_{2,0} \cap \mathcal{M C}$, which was investigated in [CS16]. Throughout this section, $\mathbf{A} \in \mathcal{S}$.

In this section our goal is to prove that 47 of the subvarieties of $\mathcal{S}$ of Bol-Moufang type are equal to $\mathcal{S} \mathcal{L}$ and hence are equal to each other. First, we present some new properties of $\mathcal{S}$ which will be useful later in this paper.

LEMMA 3.1. A satisfies:

(a) $x \rightarrow(y \rightarrow z) \approx y \rightarrow(x \rightarrow z)$

(b) $x^{\prime} \rightarrow y \approx y^{\prime} \rightarrow x$,

(c) $x \rightarrow y \approx y^{\prime} \rightarrow x^{\prime}$,

(d) $x \rightarrow y^{\prime} \approx y \rightarrow x^{\prime}$.

Proof. Let $a, b, c \in A$. Then

(a)

$$
\begin{aligned}
a \rightarrow(b \rightarrow c) & =\left(a \rightarrow b^{\prime}\right)^{\prime} \rightarrow c \quad \text { by Lemma } 2.4(\underline{\mathbb{k}}) \\
& =\left(b \rightarrow a^{\prime}\right)^{\prime} \rightarrow c \quad \text { by (MC) } \\
& =b \rightarrow(a \rightarrow c) \quad \text { by Lemma } 2.4(\underline{\mathbb{k}}) .
\end{aligned}
$$

(b)

$$
\begin{aligned}
a^{\prime} \rightarrow b & =\left(a^{\prime} \rightarrow b^{\prime \prime}\right)^{\prime \prime} & \text { by }\left(\overline{I_{2,0}}\right) \\
& =\left(b^{\prime} \rightarrow a^{\prime \prime}\right)^{\prime \prime} & \text { by }(\overline{\mathrm{MC}}) \\
& =b^{\prime} \rightarrow a & \text { by }\left(\overline{\mathrm{I}_{2,0}} .\right.
\end{aligned}
$$


(c)

$$
\begin{aligned}
a \rightarrow b & =a^{\prime \prime} \rightarrow b \quad \text { by }\left(\mathrm{I}_{2,0}\right. \\
& =b^{\prime} \rightarrow a^{\prime} \quad \text { by item (b) }
\end{aligned}
$$

(d)

$$
\begin{aligned}
a \rightarrow b^{\prime} & =a^{\prime \prime} \rightarrow b^{\prime} \quad \text { by } \quad \mathrm{I}_{2,0} \\
& =b^{\prime \prime} \rightarrow a^{\prime} \quad \text { by item (b) } \\
& =b \rightarrow a^{\prime} \quad \text { by } \quad \mathrm{I}_{2,0}
\end{aligned}
$$

LEMMA 3.2. Let $\mathbf{A} \models x \rightarrow x \approx x$. Then $\mathbf{A} \models x^{\prime} \approx x$.

Proof. Let $a \in A$. Then

$$
\begin{aligned}
a^{\prime} & =(a \rightarrow a)^{\prime} & & \text { by hypothesis } \\
& =\left(a \rightarrow a^{\prime \prime}\right)^{\prime} & & \text { by }\left(\mathrm{I}_{2,0}\right) \\
& =\left(a^{\prime} \rightarrow a^{\prime}\right)^{\prime} & & \text { by }(\underline{\mathrm{MC}}) \\
& =a^{\prime \prime} & & \text { by hypothesis } \\
& =a & & \text { by }\left(\mathrm{I}_{2,0}\right) .
\end{aligned}
$$

LEMMA 3.3. Let $\mathbf{A} \models x^{\prime} \approx x \rightarrow x$ and $\mathbf{A} \models 0^{\prime} \approx 0$. Then $\mathbf{A} \models x^{\prime} \approx x$.

Proof. Let $a \in A$. Then

$$
\begin{aligned}
a & =a^{\prime \prime} & & \\
& =(a \rightarrow a)^{\prime} & & \text { by hypothesis } \\
& =(a \rightarrow a) \rightarrow 0 & & \\
& =(a \rightarrow a) \rightarrow 0^{\prime} & & \text { by hypothesis } \\
& =\left\{\left(0^{\prime \prime} \rightarrow a\right) \rightarrow\left(a \rightarrow 0^{\prime}\right)^{\prime}\right\}^{\prime} & & \text { by }(\mathrm{I}) \\
& =\left\{(0 \rightarrow a) \rightarrow\left(a \rightarrow 0^{\prime}\right)^{\prime}\right\}^{\prime} & & \text { by }\left(\mathrm{I}_{2,0}\right) \\
& =\left\{(0 \rightarrow a) \rightarrow(a \rightarrow 0)^{\prime}\right\}^{\prime} & & \text { by hypothesis } \\
& =\left\{(0 \rightarrow a) \rightarrow a^{\prime \prime}\right\}^{\prime} & & \\
& =\{(0 \rightarrow a) \rightarrow a\}^{\prime} & & \text { by }\left(\overline{I_{2,0}}\right) \\
& =\{(a \rightarrow a) \rightarrow a\}^{\prime} & & \text { by Lemma } 2.4(\mathrm{~d}) \\
& =\left(a^{\prime} \rightarrow a\right)^{\prime} & & \text { by hypothesis } \\
& =a^{\prime} & & \text { by Lemma } 2.2(\mathrm{~d}),
\end{aligned}
$$

completing the proof.

LEMMA 3.4. Let $\mathbf{A} \models 0 \rightarrow(x \rightarrow x) \approx x \rightarrow x$. Then

$\mathbf{A} \models(x \rightarrow x) \rightarrow(y \rightarrow z) \approx((x \rightarrow x) \rightarrow y) \rightarrow z$. 
Proof. Let $a, b, c \in A$. Now,

$$
\begin{aligned}
& (a \rightarrow a) \rightarrow b^{\prime}=(0 \rightarrow(a \rightarrow a)) \rightarrow b^{\prime} \quad \text { by hypothesis } \\
& =\left(b^{\prime} \rightarrow(a \rightarrow a)\right) \rightarrow b^{\prime} \quad \text { by Lemma } 2.4 \text { (ㄷ) } \\
& =\left\{\left(b^{\prime \prime} \rightarrow b^{\prime}\right) \rightarrow\left((a \rightarrow a) \rightarrow b^{\prime}\right)^{\prime}\right\}^{\prime} \text { by (I) } \\
& =\left\{b^{\prime} \rightarrow\left((a \rightarrow a) \rightarrow b^{\prime}\right)^{\prime}\right\}^{\prime} \quad \text { by Lemma } 2.2 \text { (d) } \\
& =\left\{\left((a \rightarrow a) \rightarrow b^{\prime}\right)^{\prime \prime} \rightarrow b\right\}^{\prime} \quad \text { by Lemma 3.1 ( (b) } \\
& =\left\{\left((a \rightarrow a) \rightarrow b^{\prime}\right) \rightarrow b\right\}^{\prime} \quad \text { by }\left(\mathrm{I}_{2,0}\right) \\
& =\left\{\left((a \rightarrow a) \rightarrow 0^{\prime}\right) \rightarrow b\right\}^{\prime} \quad \text { by Lemma } 2.4 \text { (国) } \\
& =\left\{\left(0 \rightarrow(a \rightarrow a)^{\prime}\right) \rightarrow b\right\}^{\prime} \quad \text { by Lemma } 2.3(\mathrm{~b}) \\
& =\left\{\left(0 \rightarrow\left(a \rightarrow a^{\prime \prime}\right)^{\prime}\right) \rightarrow b\right\}^{\prime} \quad \text { by }\left(\mathrm{I}_{2,0}\right) \\
& =\left\{\left(0 \rightarrow\left(a^{\prime} \rightarrow a^{\prime}\right)\right) \rightarrow b\right\}^{\prime} \quad \text { by Lemma } 2.4 \text { (1) } \\
& =\left\{\left(a^{\prime} \rightarrow a^{\prime}\right) \rightarrow b\right\}^{\prime} \quad \text { by hypothesis } \\
& =\{(a \rightarrow a) \rightarrow b\}^{\prime} \quad \text { by Lemma } 3.1(\mathrm{~b}) \text {. }
\end{aligned}
$$

Therefore,

$$
\mathbf{A} \models(\mathrm{x} \rightarrow \mathrm{x}) \rightarrow \mathrm{y}^{\prime} \approx((\mathrm{x} \rightarrow \mathrm{x}) \rightarrow \mathrm{y})^{\prime}
$$

Hence,

$$
\begin{aligned}
& ((a \rightarrow a) \rightarrow b) \rightarrow c=c^{\prime} \rightarrow[(a \rightarrow a) \rightarrow b]^{\prime} \quad \text { by Lemma 3.1 (b) } \\
& =c^{\prime} \rightarrow\left[(a \rightarrow a) \rightarrow b^{\prime}\right] \text { by (1) } \\
& =(a \rightarrow a) \rightarrow\left(c^{\prime} \rightarrow b^{\prime}\right) \text { by Lemma } 3.1 \text { (国) } \\
& =(a \rightarrow a) \rightarrow(b \rightarrow c) \quad \text { by Lemma } 3.1 \text { (b) } \text {. }
\end{aligned}
$$

This completes the proof.

LEMMA 3.5. $\mathcal{B A} \subseteq \mathcal{A}_{12}$.

Proof. It is routine to check that $\mathbf{2}_{\mathrm{b}} \in \mathcal{A}_{12}$. The proof is complete since we know $\mathcal{V}\left(\mathbf{2}_{\mathbf{b}}\right)=\mathcal{B} \mathcal{A}$.

LEMMA 3.6. $\mathcal{S} \mathcal{L} \subseteq \mathcal{X}_{i j}$, for $\mathcal{X} \in\{\mathcal{A}, \mathcal{B}, \mathcal{C}, \mathcal{D}, \mathcal{E}, \mathcal{F}\}$ and for all $1 \leq i<$ $j \leq 5$.

Proof. By a routine computation, it is easy to check that $\mathbf{2}_{\mathbf{s}} \in \mathcal{X}_{i j}$ for all $1 \leq i<j \leq 5$. Then the proof is complete, in view of $\mathcal{V}\left(\mathbf{2}_{\mathbf{s}}\right)=\mathcal{S} \mathcal{L}$.

Let $\mathbf{M}$ denote the set that contains the subvarieties $\mathcal{A}_{13}, \mathcal{A}_{14}, \mathcal{A}_{15}, \mathcal{A}_{24}$, $\mathcal{A}_{34}, \mathcal{A}_{45}, \mathcal{B}_{12}, \mathcal{B}_{14}, \mathcal{B}_{15}, \mathcal{B}_{23}, \mathcal{B}_{24}, \mathcal{B}_{34}, \mathcal{B}_{35}, \mathcal{B}_{45}, \mathcal{C}_{12}, \mathcal{C}_{13}, \mathcal{C}_{14}, \mathcal{C}_{15}, \mathcal{C}_{23}, \mathcal{C}_{24}$, $\mathcal{C}_{34}, \mathcal{C}_{35}, \mathcal{C}_{45}, \mathcal{D}_{13}, \mathcal{D}_{14}, \mathcal{D}_{15}, \mathcal{D}_{23}, \mathcal{D}_{24}, \mathcal{D}_{34}, \mathcal{D}_{45}, \mathcal{E}_{12}, \mathcal{E}_{13}, \mathcal{E}_{14}, \mathcal{E}_{15}, \mathcal{E}_{23}, \mathcal{E}_{24}$, $\mathcal{E}_{34}, \mathcal{E}_{35}, \mathcal{E}_{45}, \mathcal{F}_{12}, \mathcal{F}_{14}, \mathcal{F}_{15}, \mathcal{F}_{23}, \mathcal{F}_{24}, \mathcal{F}_{34}, \mathcal{F}_{35}, \mathcal{F}_{45}$. Observe that $\mathbf{M}$ has 47 elements. 
THEOREM 3.7. If $\mathcal{X} \in \mathbf{M}$ then $\mathcal{X}=\mathcal{S} \mathcal{L}$.

Proof. In the proof below the following list of statements will be useful.

The identity $\left(\overline{\mathrm{I}_{2,0}}\right)$, Lemma 2.2 (国), Lemma 2.2 (d) , Lemma 2.4 (国), Lemma 2.4 (b) , Lemma 2.4 (国) and Lemma 3.1 (国).

Let $\mathcal{X} \in M$. In view of Lemma 3.6, it suffices to prove that $\mathcal{X} \subseteq \mathcal{S} \mathcal{L}$. In fact, by Lemma 2.1, it suffices to prove that $\mathcal{X} \models x^{\prime} \approx x$. Let $\mathbf{A} \in \mathcal{X}$ and let $a \in \mathbf{A}$.

To facilitate a uniform presentation (and to make the proof shorter), we introduce the following notation, where $x_{0}, y_{0}, z_{0} \in \mathbf{A}$ :

The notation

$$
\mathcal{X} / x_{0}, y_{0}, z_{0}
$$

denotes the following statement:

"Given an algebra $\mathbf{A} \in \mathcal{X}$, with $\mathcal{X}$ being defined by the identity $(X)$, consider $x_{0}, y_{0}, z_{0} \in \mathbf{A}$. By substituting $x:=x_{0}, y:=y_{0}$ and $z:=z_{0}$ in $(X)$ and simplifying using the list $(*)$, we obtain a proof of $\mathbf{A} \models x \rightarrow x \approx x$."

For example, suppose we want to prove that the statement " $\mathcal{A}_{24} / a, a$ ', $a$ " is true. We start with $\mathbf{A} \in \mathcal{A}_{24}$ and observe that

$$
\begin{aligned}
& a=a^{\prime} \rightarrow a \quad \text { by Lemma } 2.2 \text { (d) } \\
& =\left(a^{\prime \prime} \rightarrow a^{\prime}\right) \rightarrow a \quad \text { by Lemma } 2.2 \text { (d) } \\
& =\left(a \rightarrow a^{\prime}\right) \rightarrow a \quad \text { by }\left(\mathrm{I}_{2,0}\right) \\
& =\left(a \rightarrow\left(a \rightarrow a^{\prime}\right)\right) \rightarrow a \text { by Lemma } 2.4 \text { (国) } \\
& =a \rightarrow\left(\left(a \rightarrow a^{\prime}\right) \rightarrow a\right) \text { by }\left(A_{24}\right) \\
& =a \rightarrow\left(\left(a^{\prime \prime} \rightarrow a^{\prime}\right) \rightarrow a\right) \text { by }\left(\mathrm{I}_{2,0}\right) \\
& =a \rightarrow\left(a^{\prime} \rightarrow a\right) \quad \text { by Lemma } 2.2 \text { (d) } \\
& =a \rightarrow a \quad \text { by Lemma } 2.2 \text { (d) }
\end{aligned}
$$

Consequently,

$$
\mathbf{A} \models x \approx x \rightarrow x
$$

By Lemma 3.2 ,

$$
\mathbf{A} \models x \approx x^{\prime} .
$$

We divide the 47 varieties under consideration into several groups (again, with a view to making this proof shorter) as follows:

Firstly, we consider the varieties associated with the following statements: 

1. $\mathcal{A}_{24} / a, a^{\prime}, a$,
9. $\mathcal{B}_{45} / a, 0^{\prime}, 0$,
17. $\mathcal{D}_{23} / a, 0^{\prime}, 0^{\prime}$
2. $\mathcal{A}_{45} / a, 0,0$,
10. $\mathcal{C}_{15} / 0^{\prime}, a, 0$,
18. $\mathcal{D}_{24} / a, 0^{\prime}, 0^{\prime}$,
3. $\mathcal{B}_{12} / a^{\prime}, a, 0$,
11. $\mathcal{C}_{23} / 0^{\prime}, a, 0$,
19. $\mathcal{D}_{45} / a, 0,0$,
4. $\mathcal{B}_{14} / a, a, 0$,
12. $\mathcal{C}_{24} / a, 0, a$,
20. $\mathcal{E}_{15} / 0^{\prime}, a, 0^{\prime}$,
5. $\mathcal{B}_{23} / a^{\prime}, 0,0$,
13. $\mathcal{C}_{35} / 0^{\prime}, a, 0$,
21. $\mathcal{E}_{23} / 0^{\prime}, a, 0^{\prime}$,
6. $\mathcal{B}_{24} / a, 0^{\prime}, 0$,
14. $\mathcal{C}_{45} / a, 0, a$,
22. $\mathcal{E}_{34} / 0^{\prime}, a, 0^{\prime}$,
7. $\mathcal{B}_{34} / 0^{\prime}, a, a$,
15. $\mathcal{D}_{13} / a, a^{\prime}, a^{\prime}$,
23. $\mathcal{E}_{35} / 0^{\prime}, a, 0^{\prime}$,
8. $\mathcal{B}_{35} / 0^{\prime}, a, a$,
16. $\mathcal{D}_{14} / a, a, a^{\prime}$,
24. $\mathcal{F}_{24} / a, a, 0$.

It is routine to verify that each of the above statements is true, from which it follows that, in each case, $\mathcal{X} \models x \rightarrow x \approx x$. Then, applying Lemma 3.2, we get that $\mathcal{X} \models x^{\prime} \approx x$.

The notation

$$
\mathcal{X} / x_{0}, y_{0}, z_{0} / x_{1}, y_{1}, z_{1} / p \approx q
$$

is an abbreviation for the following statement:

"Given an algebra $\mathbf{A} \in \mathcal{X}$, with $\mathcal{X}$ being defined by the identity $(X)$, consider $x_{0}, y_{0}, z_{0} \in \mathbf{A}$. By substituting $x:=x_{0}, y:=y_{0}, z:=z_{0}$, and simplifying $(\mathrm{X})$ using (the appropriate lemmas from) the list $(*)$, we obtain a proof of $\mathbf{A}=0^{\prime} \approx 0$. Then, by substituting $x:=x_{1}, y:=y_{1}, z:=z_{1}$ in the identity $(\mathrm{X})$ and simplifying it, using $0^{\prime}=0$ and the list $(*)$, we obtain a proof of $\mathbf{A} \models p \approx q . "$

Secondly, consider the varieties associated with the following statements:
1. $\mathcal{D}_{15} / 0,0,0^{\prime} / 0, a, a / x \rightarrow x \approx x$,
4. $\mathcal{E}_{14} / 0,0,0 / a, a^{\prime}, 0 / x \rightarrow x \approx x$,
2. $\mathcal{D}_{34} / 0,0,0 / 0^{\prime}, a, a / x \rightarrow x \approx x$,
5. $\mathcal{E}_{24} / 0,0,0 / a, 0, a / x \rightarrow x \approx x$,
3. $\mathcal{E}_{13} / 0,0,0^{\prime} / a^{\prime}, 0, a^{\prime} / x \rightarrow x \approx x$,
6. $\mathcal{E}_{45} / 0,0,0 / a, 0, a / x \rightarrow x \approx x$.

It is straightforward to verify that each of the above statements is true. Hence, it follows that in each case $\mathcal{X} \models x \rightarrow x \approx x$. Then, applying Lemma 3.2. we get that $\mathcal{X} \models x^{\prime} \approx x$. 
Thirdly, consider the varieties associated with the following statements:
1. $\mathcal{A}_{14} / 0,0,0 / a, 0^{\prime}, 0 / x^{\prime} \approx x$,
4. $\mathcal{F}_{34} / 0^{\prime}, 0,0 / a, a, 0 / x^{\prime} \approx x$,
2. $\mathcal{F}_{14} / 0,0,0 / a, 0,0 / x^{\prime} \approx x$,
5. $\mathcal{F}_{35} / 0^{\prime}, 0,0 / 0^{\prime}, a, 0 / x^{\prime} \approx x$
3. $\mathcal{F}_{23} / 0^{\prime}, 0,0 / a, 0,0 / x^{\prime} \approx x$,
6. $\mathcal{F}_{45} / 0,0,0 / a, 0,0 / x^{\prime} \approx x$.

It is easy to verify that the above statements are true. Hence, it follows in each of the above cases that $\mathcal{X} \models x^{\prime} \approx x$.

Lastly, consider the varieties associated with the following statements:

1. $\mathcal{A}_{15} / 0,0^{\prime}, 0 / a, 0,0 / x^{\prime} \approx x \rightarrow x$,

2. $\mathcal{A}_{34} / 0,0,0 / a, 0,0 / x^{\prime} \approx x \rightarrow x$,

3. $\mathcal{F}_{15} / 0^{\prime}, 0,0 / a, a^{\prime}, 0 / x^{\prime} \approx x \rightarrow x$.

It is clear that each of the above statements is true. Hence in each case $\mathcal{X} \models x^{\prime} \approx x \rightarrow x$. Then, applying Lemma 3.3, we get that $\mathcal{X} \models x^{\prime} \approx x$.

Thus, the varieties still left to consider are $\mathcal{A}_{13}, \mathcal{B}_{15}, \mathcal{C}_{12}, \mathcal{C}_{13}, \mathcal{C}_{14}, \mathcal{C}_{34}$, $\mathcal{E}_{12}$ and $\mathcal{F}_{12}$.

From Lemma 3.1 (国) we can easily verify that $\mathcal{C}_{13}=\mathcal{B}_{12}, \mathcal{C}_{14}=\mathcal{B}_{14}$, $\mathcal{C}_{34}=\mathcal{B}_{24}, \mathcal{E}_{12}=\mathcal{D}_{13}$, and $\mathcal{F}_{12}=\mathcal{E}_{13}$, from which we have, in view of earlier conclusions that $\mathcal{C}_{13}=\mathcal{C}_{14}=\mathcal{C}_{34}=\mathcal{E}_{12}=\mathcal{E}_{14}=\mathcal{F}_{12}=\mathcal{S} \mathcal{L}$.

Now, we are left with $\mathcal{A}_{13}, \mathcal{B}_{15}$, and $\mathcal{C}_{12}$ to consider. But, it can be easily seen, using Lemma 3.1, that $\mathcal{C}_{12}=\mathcal{A}_{13}$. Thus, it only remains to verify that $\mathcal{A}_{13}=\mathcal{S} \mathcal{L}$ and $\mathcal{B}_{15}=\mathcal{S} \mathcal{L}$.

First, we show that $\mathcal{A}_{13}=\mathcal{S} \mathcal{L}$. Let $\mathbf{A} \in \mathcal{A}_{13}$ and let $a \in A$. Then

$$
\begin{aligned}
a & =0^{\prime} \rightarrow a & & \text { by Lemma } 2.2 \text { (国) } \\
& =(0 \rightarrow 0) \rightarrow a & & \\
& =(0 \rightarrow 0) \rightarrow\left(a^{\prime} \rightarrow a\right) & & \text { by Lemma } 2.2 \text { (d) } \\
& =0 \rightarrow\left(0 \rightarrow\left(a^{\prime} \rightarrow a\right)\right) & & \text { by }\left(A_{13}\right) \\
& =0 \rightarrow(0 \rightarrow a) & & \text { by Lemma } 2.2 \text { (dd) } \\
& =0 \rightarrow a & & \text { by Lemma } 2.4 \text { (迆). }
\end{aligned}
$$

Hence

$$
\mathbf{A} \models x \approx 0 \rightarrow x
$$


Therefore

$$
\begin{aligned}
& a=a^{\prime} \rightarrow a \quad \text { by Lemma } 2.2 \text { (d) } \\
& =a^{\prime} \rightarrow\left(a^{\prime} \rightarrow a\right) \quad \text { by Lemma } 2.4 \text { (国) } \\
& =a^{\prime} \rightarrow\left(a^{\prime} \rightarrow a^{\prime \prime}\right) \quad \text { by }\left(\mathrm{I}_{2,0}\right) \\
& =a^{\prime} \rightarrow\left(a^{\prime} \rightarrow\left(a^{\prime} \rightarrow 0\right)\right) \\
& =\left(a^{\prime} \rightarrow a^{\prime}\right) \rightarrow\left(a^{\prime} \rightarrow 0\right) \text { by }\left(A_{13}\right) \\
& =\left(a^{\prime} \rightarrow a^{\prime}\right) \rightarrow a^{\prime \prime} \\
& =\left(a^{\prime} \rightarrow 0^{\prime}\right) \rightarrow a^{\prime \prime} \quad \text { by Lemma } 2.4 \text { (国) } \\
& =\left(0 \rightarrow a^{\prime \prime}\right) \rightarrow a^{\prime \prime} \quad \text { by Lemma } 2.3 \text { (b) } \\
& =(0 \rightarrow a) \rightarrow a \quad \text { by }\left(\mathrm{I}_{2,0}\right) \\
& =a \rightarrow a \quad \text { by (2). }
\end{aligned}
$$

Using Lemma 3.2 we have that $\mathbf{A} \in \mathcal{S} \mathcal{L}$, hence $\mathcal{A}_{13} \subseteq \mathcal{S L}$, implying $\mathcal{A}_{13}=\mathcal{S} \mathcal{L}$.

Next, we prove that $\mathcal{B}_{15}=\mathcal{S} \mathcal{L}$. Let $\mathbf{A} \in \mathcal{B}_{15}$ and let $a \in A$. Then

$$
\begin{aligned}
a & =a^{\prime \prime} & & \text { by }\left(\overline{I_{2,0}}\right) \\
& =\left(a^{\prime \prime} \rightarrow a^{\prime}\right)^{\prime} & & \text { by Lemma } 2.2(\mathrm{~d}) \\
& =\left(\left(a^{\prime} \rightarrow 0\right) \rightarrow a^{\prime}\right)^{\prime} & & \\
& =\left(\left(a^{\prime} \rightarrow 0\right) \rightarrow a^{\prime}\right) \rightarrow 0 & & \\
& =a^{\prime} \rightarrow\left(0 \rightarrow\left(a^{\prime} \rightarrow 0\right)\right) & & \text { by }\left(B_{15}\right) \\
& =a^{\prime} \rightarrow\left(0 \rightarrow a^{\prime \prime}\right) & & \\
& =a^{\prime} \rightarrow(0 \rightarrow a) & & \text { by }\left(I_{2,0}\right) \\
& =0 \rightarrow\left(a^{\prime} \rightarrow a\right) & & \text { by Lemma } 3.1(\text { (ad) } \\
& =0 \rightarrow a & & \text { by Lemma } 2.2\left(\frac{\mathrm{d} d}{\mathrm{~d}}\right) .
\end{aligned}
$$

Hence

$$
\begin{aligned}
a & =a^{\prime \prime} & & \text { by }\left(\overline{I_{2,0}}\right) \\
& =\left(a^{\prime \prime} \rightarrow a^{\prime}\right)^{\prime} & & \text { by Lemma } 2.2(\mathrm{~d}) \\
& =\left(a \rightarrow a^{\prime}\right)^{\prime} & & \text { by }\left(\bar{I}_{2,0}\right) \\
& =(a \rightarrow(a \rightarrow 0))^{\prime} & & \\
& =(a \rightarrow(a \rightarrow(a \rightarrow 0)))^{\prime} & & \text { by Lemma } 2.4(\mathbb{G}) \\
& =((a \rightarrow a) \rightarrow a)^{\prime \prime} & & \text { by }\left(B_{15}\right) \\
& =(a \rightarrow a) \rightarrow a & & \text { by }\left(\overline{I_{2,0}}\right) \\
& =(0 \rightarrow a) \rightarrow a & & \text { by Lemma } 2.4(\text { (c) }) \\
& =a \rightarrow a & & \text { by previous calculus. }
\end{aligned}
$$

Thus,

$$
\mathbf{A} \models x \approx x \rightarrow x .
$$

By Lemma 3.2 we have that $\mathbf{A} \models x^{\prime} \approx x$, implying that $\mathcal{B}_{15} \subseteq \mathcal{S} \mathcal{L}$. So, $\mathcal{B}_{15}=\mathcal{S} \mathcal{L}$, completing the proof. 
The following corollary is immediate from the above theorem.

COROLLARY 3.8. $M=\{\mathcal{S} \mathcal{L}\}$.

Thus, there are 47 Bol-Moufang subvarieties each of which is equal to $\mathcal{S L}$; and hence they are equal to each other.

In order to be able to compare the remaining (possibly distinct) subvarieties with each other, the following lemmas will be useful.

LEMMA 3.9. Let $\mathbf{A} \in \mathcal{A}_{23}$. Then $\mathbf{A}$ satisfies the identities:

(a) $0 \rightarrow x \approx x$

(b) $(x \rightarrow x) \rightarrow y \approx x \rightarrow\left(\left(x \rightarrow 0^{\prime}\right) \rightarrow y\right)$,

(c) $(x \rightarrow x) \rightarrow y \approx x \rightarrow\left(x \rightarrow y^{\prime}\right)^{\prime}$.

Proof. (a) Let $a \in A$. Then

$$
\begin{aligned}
a & =0^{\prime} \rightarrow a & & \text { by Lemma } 2.2(\text { (国) } \\
& =(0 \rightarrow 0) \rightarrow a & & \\
& =(0 \rightarrow 0) \rightarrow a^{\prime \prime} & & \text { by }\left(\mathrm{I}_{2,0}\right) \\
& =(0 \rightarrow 0) \rightarrow\left(a^{\prime} \rightarrow 0\right) & & \\
& =0 \rightarrow\left(0 \rightarrow a^{\prime}\right)^{\prime} & & \text { by }\left(A_{23}\right) \\
& =0 \rightarrow a^{\prime \prime} & & \text { by Lemma } 2.4(\mathbb{f}) \\
& =0 \rightarrow a & & \text { by }\left(\mathrm{I}_{2,0}\right) .
\end{aligned}
$$

(b) Let $a, b \in A$. Then, using $\left(A_{23}\right)$ and Lemma 2.2 (国), we have that $a \rightarrow\left(\left(a \rightarrow 0^{\prime}\right) \rightarrow b\right)=(a \rightarrow a) \rightarrow\left(0^{\prime} \rightarrow b\right)=(a \rightarrow a) \rightarrow b$.

(c) Let $a, b \in A$. By $\left(A_{23}\right)$ we have that $(a \rightarrow a) \rightarrow b=(a \rightarrow a) \rightarrow b^{\prime \prime}=$ $(a \rightarrow a) \rightarrow\left(b^{\prime} \rightarrow 0\right)=a \rightarrow\left(a \rightarrow b^{\prime}\right)^{\prime}$.

LEMMA 3.10. We have

(1) Let $\mathbf{A} \in \mathcal{A}_{25} \cup \mathcal{C}_{25} \cup \mathcal{D}_{25} \cup \mathcal{E}_{25}$. Then $\mathbf{A}$ satisfies the identities

(a) $0 \rightarrow x \approx x$

(b) $(x \rightarrow y)^{\prime} \approx x^{\prime} \rightarrow y^{\prime}$.

(2) Let $\mathbf{A} \in \mathcal{A}_{25}$. Then $\mathbf{A} \models x \rightarrow\left(y \rightarrow\left(y^{\prime} \rightarrow z\right)\right) \approx y \rightarrow((y \rightarrow x) \rightarrow z)$. 
Proof. (1) (a) Let $\mathbf{A} \in \mathcal{A}_{25}$ and $a \in A$. Then

$$
\begin{aligned}
a & =a^{\prime \prime} & & \text { by }\left(\underline{I}_{2,0}\right) \\
& =a^{\prime} \rightarrow 0 & & \\
& =\left(0^{\prime} \rightarrow a^{\prime}\right) \rightarrow 0 & & \text { by Lemma } 2.2(\underline{a}) \\
& =\left((0 \rightarrow 0) \rightarrow a^{\prime}\right) \rightarrow 0 & & \\
& =0 \rightarrow\left(\left(0 \rightarrow a^{\prime}\right) \rightarrow 0\right) & & \text { by }\left(A_{25}\right) \\
& =0 \rightarrow a^{\prime \prime} & & \text { by Lemma } 2.4(\mathbb{f}) \\
& =0 \rightarrow a & & \text { by }\left(I_{2,0}\right) .
\end{aligned}
$$

Let $\mathbf{A} \in \mathcal{C}_{25}$ and $a \in A$. Then

$$
\begin{aligned}
0 \rightarrow a & =0 \rightarrow\left(0^{\prime} \rightarrow a\right) & & \text { by Lemma } 2.2 \text { (回) } \\
& =0 \rightarrow\left(\left(0^{\prime} \rightarrow 0^{\prime}\right) \rightarrow a\right) & & \text { by Lemma } 2.2 \text { (国) } \\
& =\left(\left(0 \rightarrow 0^{\prime}\right) \rightarrow 0^{\prime}\right) \rightarrow a & & \text { by }\left(C_{25}\right) \\
& =\left(\left(0^{\prime \prime} \rightarrow 0^{\prime}\right) \rightarrow 0^{\prime}\right) \rightarrow a & & \text { by } 0 \approx 0^{\prime \prime} \\
& =\left(0^{\prime} \rightarrow 0^{\prime}\right) \rightarrow a & & \text { by Lemma } 2.2 \text { (迆) } \\
& =0^{\prime} \rightarrow a & & \text { by Lemma } 2.2 \text { (国) } \\
& =a & & \text { by Lemma } 2.2 \text { (回). }
\end{aligned}
$$

Let $\mathbf{A} \in \mathcal{D}_{25}$ and $a \in A$. Then

$$
\begin{aligned}
0 \rightarrow a & =0 \rightarrow a^{\prime \prime} & & \text { by }\left(\mathrm{I}_{2,0}\right) \\
& =0 \rightarrow\left(0 \rightarrow a^{\prime}\right)^{\prime} & & \text { by Lemma } 2.4(\mathbb{f}) \\
& =0 \rightarrow\left(\left(0 \rightarrow a^{\prime}\right) \rightarrow 0\right) & & \\
& =\left((0 \rightarrow 0) \rightarrow a^{\prime}\right) \rightarrow 0 & & \text { by }\left(D_{25}\right) \\
& =\left(0^{\prime} \rightarrow a^{\prime}\right) \rightarrow 0 & & \\
& =a^{\prime} \rightarrow 0 & & \text { by Lemma } 2.2 \text { (回) } \\
& =a & & \text { by }\left(\mathrm{I}_{2,0} .\right.
\end{aligned}
$$

If $\mathbf{A} \in \mathcal{E}_{25}$, then the proof is analogous to that of the preceding case. Just replace $\left(D_{25}\right)$ with $\left(E_{25}\right)$.

(b) By (1a), $\mathbf{A} \models x \approx 0 \rightarrow x$. Then by Lemma 2.5, we have

$$
\mathbf{A} \models(x \rightarrow y)^{\prime} \approx x^{\prime} \rightarrow y^{\prime}
$$


(2) Let $a, b, c \in A$. Then

$$
\begin{aligned}
& a \rightarrow\left(b \rightarrow\left(b^{\prime} \rightarrow c\right)\right)=a \rightarrow\left((b \rightarrow b)^{\prime} \rightarrow c\right) \text { by }\left(A_{25}\right) \\
& =a \rightarrow\left(c^{\prime} \rightarrow(b \rightarrow b)\right) \text { by Lemma } 3.1 \text { (b) } \\
& =a \rightarrow\left(b \rightarrow\left(c^{\prime} \rightarrow b\right)\right) \text { by Lemma } 3.1 \text { (国) } \\
& =b \rightarrow\left(a \rightarrow\left(c^{\prime} \rightarrow b\right)\right) \text { by Lemma 3.1 (国) } \\
& =b \rightarrow\left(c^{\prime} \rightarrow(a \rightarrow b)\right) \text { by Lemma } 3.1 \text { (国) } \\
& =b \rightarrow\left((a \rightarrow b)^{\prime} \rightarrow c\right) \text { by Lemma } 3.1 \text { (BD) } \\
& =b \rightarrow\left(\left(a^{\prime} \rightarrow b^{\prime}\right) \rightarrow c\right) \text { by (1b) } \\
& =b \rightarrow((b \rightarrow a) \rightarrow c) \text { by Lemma 3.1 (ㄷ) } \text {. }
\end{aligned}
$$

This completes the proof.

LEMMA 3.11. Let $\mathbf{A} \in \mathcal{A}_{12} \cup \mathcal{D}_{12} \cup \mathcal{D}_{35}$ then $\mathbf{A}$ satisfies the identities

(a) $0 \rightarrow x^{\prime} \approx x \rightarrow x \approx 0 \rightarrow x$,

(b) $0 \rightarrow(x \rightarrow y) \approx 0 \rightarrow(y \rightarrow x)$,

(c) $0 \rightarrow(x \rightarrow(y \rightarrow z)) \approx 0 \rightarrow((x \rightarrow y) \rightarrow z)$.

Proof. Let $a, b, c \in A$.

(a) Suppose $\mathbf{A} \in \mathcal{A}_{12}$. Then

$$
\begin{aligned}
0 \rightarrow a^{\prime} & =a \rightarrow 0^{\prime} & & \text { by Lemma } 2.3(\mathrm{~b}) \\
& =a \rightarrow\left(a \rightarrow 0^{\prime}\right) & & \text { by Lemma } 2.4 \text { (远) } \\
& =a \rightarrow(a \rightarrow(0 \rightarrow 0)) & & \\
& =a \rightarrow((a \rightarrow 0) \rightarrow 0) & & \text { by }\left(A_{12}\right) \\
& =a \rightarrow a^{\prime \prime} & & \\
& =a \rightarrow a & & \text { by }\left(\mathrm{I}_{2,0}\right) .
\end{aligned}
$$

Thus, we have

$$
\mathbf{A} \models 0 \rightarrow x^{\prime} \approx x \rightarrow x .
$$

Hence,

$$
\begin{aligned}
0 \rightarrow a & =0 \rightarrow a^{\prime \prime} \quad \text { by }\left(\overline{\mathrm{I}_{2,0}}\right) \\
& =a^{\prime} \rightarrow a^{\prime} \quad \text { by }(\underline{3}) \\
& =a \rightarrow a \quad \text { by Lemma } 3.1(\text { (C) }) .
\end{aligned}
$$

Consequently,

$$
\mathbf{A} \models 0 \rightarrow x \approx x \rightarrow x .
$$

Next, assume that $\mathbf{A} \in \mathcal{D}_{12}$. Then 


$$
\begin{aligned}
a \rightarrow a & =a \rightarrow\left(0^{\prime} \rightarrow a\right) & & \text { by Lemma } 2.2 \text { (国) } \\
& =a \rightarrow((0 \rightarrow 0) \rightarrow a) & & \\
& =a \rightarrow(0 \rightarrow(0 \rightarrow a)) & & \text { by }\left(D_{12}\right) \\
& =0 \rightarrow(0 \rightarrow(a \rightarrow a)) & & \text { by Lemma } 3.1 \text { (国) twice } \\
& =0 \rightarrow(a \rightarrow a) & & \text { by Lemma } 2.4 \text { (国) } \\
& =0 \rightarrow\left(a^{\prime \prime} \rightarrow a^{\prime \prime}\right) & & \text { by }\left(\mathrm{I}_{2,0}\right) \\
& =0 \rightarrow\left(a^{\prime} \rightarrow a^{\prime}\right)^{\prime} & & \text { by Lemma 2.4 (il) } \\
& =0 \rightarrow\left(\left(a^{\prime} \rightarrow a^{\prime}\right) \rightarrow 0\right) & & \\
& =0 \rightarrow\left(a^{\prime} \rightarrow\left(a^{\prime} \rightarrow 0\right)\right) & & \text { by }\left(D_{12}\right) \\
& =0 \rightarrow\left(a^{\prime} \rightarrow a\right) & & \text { by }\left(\mathrm{I}_{2,0}\right) \\
& =0 \rightarrow a & & \text { by Lemma } 2.2 \text { (d) } .
\end{aligned}
$$

Hence

$$
\mathbf{A} \models 0 \rightarrow x \approx x \rightarrow x .
$$

Now,

$$
\begin{aligned}
0 \rightarrow a^{\prime} & =a^{\prime} \rightarrow a^{\prime} \quad \text { by (14) } \\
& =a \rightarrow a \quad \text { by Lemma } 3.1 \text { (IC). }
\end{aligned}
$$

Consequently,

$$
\mathbf{A} \models 0 \rightarrow x^{\prime} \approx x \rightarrow x .
$$

Finally, assume that $\mathbf{A} \in \mathcal{D}_{35}$. Then

$$
\begin{aligned}
0 \rightarrow a & =a^{\prime} \rightarrow(0 \rightarrow a) & & \text { by Lemma 2.4 (j]) } \\
& =(a \rightarrow 0) \rightarrow(0 \rightarrow a) & & \\
& =((a \rightarrow 0) \rightarrow 0) \rightarrow a & & \text { by }\left(D_{35}\right) \\
& =a^{\prime \prime} \rightarrow a & & \\
& =a \rightarrow a & & \text { by }\left(\mathrm{I}_{2,0}\right) .
\end{aligned}
$$

Hence

$$
\begin{aligned}
& \mathbf{A} \models 0 \rightarrow x \approx x \rightarrow x . \\
0 \rightarrow a^{\prime} & =a^{\prime} \rightarrow a^{\prime} \quad \text { by (15) } \\
& =a \rightarrow a \quad \text { by Lemma } 3.1 \text { (IC). }
\end{aligned}
$$

Consequently,

$$
\mathbf{A} \models 0 \rightarrow x^{\prime} \approx x \rightarrow x .
$$

(b) Observe that

$$
\begin{aligned}
& 0 \rightarrow(a \rightarrow b)=0 \rightarrow\left(b^{\prime} \rightarrow a^{\prime}\right) \quad \text { by Lemma } 3.1 \text { (IC) } \\
& =\left(0 \rightarrow b^{\prime}\right) \rightarrow\left(0 \rightarrow a^{\prime}\right) \text { by Lemma } 2.4 \text { (h) and (d) } \\
& =(0 \rightarrow b) \rightarrow(0 \rightarrow a) \text { by (国) } \\
& =0 \rightarrow(b \rightarrow a) \quad \text { by Lemma } 2.4(\underline{\mathrm{h}}) \text { and }(\underline{\mathrm{d}}) \text {. }
\end{aligned}
$$


(c)

$$
\begin{aligned}
& 0 \rightarrow(b \rightarrow(c \rightarrow a))=(0 \rightarrow b) \rightarrow(0 \rightarrow(c \rightarrow a)) \\
& \text { by Lemma } 2.4(\mathrm{~h}) \text { and (d) } \\
& =(0 \rightarrow b) \rightarrow(0 \rightarrow(a \rightarrow c)) \quad \text { by }(\underline{\mathrm{b}}) \\
& =0 \rightarrow(b \rightarrow(a \rightarrow c)) \\
& \text { by Lemma } 2.4(\underline{h}) \text { and (d) } \\
& =0 \rightarrow(a \rightarrow(b \rightarrow c)) \quad \text { by Lemma } 3.1 \text { (国) } \\
& =0 \rightarrow((b \rightarrow c) \rightarrow a) \quad \text { by (b). }
\end{aligned}
$$

This completes the proof.

\section{Distinct Varieties of Symmetric $\mathcal{I}$-zroupoids of Bol-Moufang type}

Recall that 47 of the 60 subvarieties of $\mathcal{S}$ were shown to be equal to the variety $\mathcal{S} \mathcal{L}$. In this section we will investigate the relationships among the remaining 13 varieties: $\mathcal{A}_{12}, \mathcal{A}_{23}, \mathcal{A}_{25}, \mathcal{A}_{35}, \mathcal{B}_{13}, \mathcal{B}_{25}, \mathcal{C}_{25}, \mathcal{D}_{12}, \mathcal{D}_{25}, \mathcal{D}_{35}, \mathcal{E}_{25}, \mathcal{F}_{13}$ and $\mathcal{F}_{25}$. We still need to determine which of these are distinct from each other. The following theorem throws more light on this issue.

THEOREM 4.1. We have

(a) $\mathcal{A}_{23}=\mathcal{A}_{25}=\mathcal{C}_{25}=\mathcal{D}_{25}=\mathcal{E}_{25}$,

(b) $\mathcal{A}_{12}=\mathcal{B}_{13}=\mathcal{D}_{12}=\mathcal{D}_{35}=\mathcal{F}_{13}$,

(c) $\mathcal{A}_{35}=\mathcal{F}_{25}$,

(d) $\mathcal{B}_{25}=\mathcal{S}$.

Proof. (a) Let $\mathbf{A} \in \mathcal{A}_{23}$ and $a, b, c \in A$. We have that

$$
\begin{aligned}
c^{\prime} \rightarrow(a \rightarrow(b \rightarrow a)) & =a \rightarrow\left(c^{\prime} \rightarrow(b \rightarrow a)\right) & \text { by Lemma 3.1 (回) } \\
& =a \rightarrow\left(b \rightarrow\left(c^{\prime} \rightarrow a\right)\right) & \text { by Lemma 3.1 (回) } \\
& =a \rightarrow\left(b \rightarrow\left(a^{\prime} \rightarrow c\right)\right) & \text { by Lemma 3.1 (殒) } \\
& =a \rightarrow\left(a^{\prime} \rightarrow(b \rightarrow c)\right) . & \text { by Lemma 3.1 (回). }
\end{aligned}
$$

Hence $\mathbf{A}$ satisfies the identity

$$
z^{\prime} \rightarrow(x \rightarrow(y \rightarrow x)) \approx x \rightarrow\left(x^{\prime} \rightarrow(y \rightarrow z)\right) .
$$


Observe

$$
\begin{aligned}
& \left(a \rightarrow\left(a \rightarrow b^{\prime}\right)^{\prime}\right)^{\prime}=\left(\left(a \rightarrow b^{\prime}\right) \rightarrow a^{\prime}\right)^{\prime} \\
& =\left(\left(a^{\prime \prime} \rightarrow a\right) \rightarrow\left(b^{\prime} \rightarrow a^{\prime}\right)^{\prime}\right)^{\prime \prime} \\
& =(a \rightarrow a) \rightarrow\left(b^{\prime} \rightarrow a^{\prime}\right)^{\prime} \\
& =(a \rightarrow a) \rightarrow\left((b \rightarrow 0) \rightarrow a^{\prime}\right)^{\prime} \\
& =(a \rightarrow a) \rightarrow\left(\left(a^{\prime \prime} \rightarrow b\right) \rightarrow\left(0 \rightarrow a^{\prime}\right)^{\prime}\right)^{\prime \prime} \quad \text { by }(\mathrm{I}) \\
& =(a \rightarrow a) \rightarrow\left((a \rightarrow b) \rightarrow\left(0 \rightarrow a^{\prime}\right)^{\prime}\right) \quad \text { by }\left(\bar{I}_{2,0}\right) \\
& =(a \rightarrow a) \rightarrow\left((a \rightarrow b) \rightarrow a^{\prime \prime}\right) \quad \text { by Lemma 3.9 (a) } \\
& =(a \rightarrow a) \rightarrow((a \rightarrow b) \rightarrow a) \quad \text { by }\left(I_{2,0}\right) \\
& =(a \rightarrow a) \rightarrow((0 \rightarrow b) \rightarrow a) \quad \text { by Lemma } 2.4 \text { (c) } \\
& =(a \rightarrow a) \rightarrow(b \rightarrow a) \\
& =a \rightarrow((a \rightarrow b) \rightarrow a) \\
& =a \rightarrow((0 \rightarrow b) \rightarrow a) \\
& =a \rightarrow(b \rightarrow a)
\end{aligned}
$$

Hence, using (6), we have that $\mathbf{A}$ satisfies

$$
z^{\prime} \rightarrow\left(x \rightarrow\left(x \rightarrow y^{\prime}\right)^{\prime}\right)^{\prime} \approx x \rightarrow\left(x^{\prime} \rightarrow(y \rightarrow z)\right) .
$$

Therefore, by Lemma 3.9 (国),

$$
\mathbf{A} \models z^{\prime} \rightarrow\left(x \rightarrow\left(x \rightarrow y^{\prime}\right)^{\prime}\right)^{\prime} \approx x \rightarrow\left(\left(0 \rightarrow x^{\prime}\right) \rightarrow(y \rightarrow z)\right) .
$$

From

$$
\begin{aligned}
a \rightarrow\left(\left(0 \rightarrow a^{\prime}\right) \rightarrow(b \rightarrow c)\right) & =a \rightarrow\left(\left(a \rightarrow 0^{\prime}\right) \rightarrow(b \rightarrow c)\right) & & \text { by Lemma 2.3 (b) } \\
& =(a \rightarrow a \rightarrow(b \rightarrow c) & & \text { by Lemma 3.9 (b) } \\
& =a \rightarrow((a \rightarrow b) \rightarrow c) & & \text { by }\left(A_{23}\right),
\end{aligned}
$$

and the equation (7), we obtain

$$
\mathbf{A} \models z^{\prime} \rightarrow\left(x \rightarrow\left(x \rightarrow y^{\prime}\right)^{\prime}\right)^{\prime} \approx x \rightarrow((x \rightarrow y) \rightarrow z) .
$$

Finally,

$$
\begin{aligned}
a \rightarrow((a \rightarrow b) \rightarrow c) & =c^{\prime} \rightarrow\left(a \rightarrow\left(a \rightarrow b^{\prime}\right)^{\prime}\right)^{\prime} & \text { by (8) } \\
& =c^{\prime} \rightarrow((a \rightarrow a) \rightarrow b)^{\prime} & \text { by Lemma } 3.9 \text { (IC) } \\
& =((a \rightarrow a) \rightarrow b) \rightarrow c & \text { by Lemma 3.1 (ㄷ) }
\end{aligned}
$$

proving that the identity $\left(A_{25}\right)$ holds in $\mathbf{A}$. Therefore, we have that

$$
\mathcal{A}_{23} \subseteq \mathcal{A}_{25}
$$


Let $\mathbf{A} \in \mathcal{A}_{25}$ and $a, b, c \in A$. Then

$$
\begin{aligned}
& ((a \rightarrow b) \rightarrow b) \rightarrow c=(((a \rightarrow b) \rightarrow b) \rightarrow c)^{\prime \prime} \quad \text { by }\left(\mathrm{I}_{2,0}\right) \\
& =\left(c^{\prime} \rightarrow((a \rightarrow b) \rightarrow b)^{\prime}\right)^{\prime \prime} \quad \text { by Lemma } 3.1 \text { (드 } \\
& =\left(c^{\prime} \rightarrow\left(b^{\prime} \rightarrow(a \rightarrow b)^{\prime}\right)^{\prime}\right)^{\prime \prime} \text { by Lemma } 3.1 \text { (ㄷ) } \\
& =\left(c^{\prime} \rightarrow(b \rightarrow(a \rightarrow b))\right)^{\prime \prime} \quad \text { by Lemma } 3.10 \text { (1b) } \\
& =\left(c^{\prime \prime} \rightarrow(b \rightarrow(a \rightarrow b))^{\prime}\right)^{\prime} \quad \text { by Lemma } 3.10 \text { (16) } \\
& =\left(c \rightarrow(b \rightarrow(a \rightarrow b))^{\prime}\right)^{\prime} \quad \text { by }\left(\overline{\mathrm{I}_{2,0}}\right) \\
& =\left(c \rightarrow\left(b \rightarrow\left(b^{\prime} \rightarrow a^{\prime}\right)\right)^{\prime}\right)^{\prime} \quad \text { by Lemma } 3.1 \text { (드 } \\
& =\left(c \rightarrow\left(b^{\prime} \rightarrow\left(b^{\prime} \rightarrow a^{\prime}\right)^{\prime}\right)\right)^{\prime} \quad \text { by Lemma } 3.10 \text { (1b) } \\
& =\left(c \rightarrow\left(b^{\prime} \rightarrow(b \rightarrow a)\right)\right)^{\prime} \quad \text { by Lemma } 3.10 \text { (1b) } \\
& =\left(c \rightarrow\left(b \rightarrow\left(b^{\prime} \rightarrow a\right)\right)\right)^{\prime} \quad \text { by Lemma } 3.1 \text { (国) } \\
& =(b \rightarrow((b \rightarrow c) \rightarrow a))^{\prime} \quad \text { by Lemma 3.10 (2) } \\
& =(((b \rightarrow b) \rightarrow c) \rightarrow a)^{\prime} \quad \text { by }\left(A_{25}\right) \\
& =\left(a^{\prime} \rightarrow((b \rightarrow b) \rightarrow c)^{\prime}\right)^{\prime} \quad \text { by Lemma } 3.1 \text { (c) } \\
& =a \rightarrow((b \rightarrow b) \rightarrow c) \quad \text { by Lemma } 3.10 \text { (1b). }
\end{aligned}
$$

Therefore, we have that

$$
\mathcal{A}_{25} \subseteq \mathcal{C}_{25}
$$


Let $\mathbf{A} \in \mathcal{C}_{25}$ and $a, b, c \in A$. We have that

$$
\begin{aligned}
& ((a \rightarrow b) \rightarrow c) \rightarrow a=((a \rightarrow b) \rightarrow c)^{\prime \prime} \rightarrow a \quad \text { by }\left(\overline{\mathrm{I}_{2,0}}\right) \\
& =\left((a \rightarrow b)^{\prime} \rightarrow c^{\prime}\right)^{\prime} \rightarrow a \text { by Lemma 3.10 (1b) } \\
& =\left(\left(a^{\prime} \rightarrow b^{\prime}\right) \rightarrow c^{\prime}\right)^{\prime} \rightarrow a \text { by Lemma 3.10 (1b) } \\
& =a^{\prime} \rightarrow\left(\left(a^{\prime} \rightarrow b^{\prime}\right) \rightarrow c^{\prime}\right) \text { by Lemma 3.1 (b) } \\
& =a^{\prime} \rightarrow\left(c \rightarrow\left(a^{\prime} \rightarrow b^{\prime}\right)^{\prime}\right) \text { by Lemma 3.1 (dd) } \\
& =c \rightarrow\left(a^{\prime} \rightarrow\left(a^{\prime} \rightarrow b^{\prime}\right)^{\prime}\right) \text { by Lemma } 3.1 \text { (a) } \\
& =c \rightarrow\left(a^{\prime} \rightarrow(a \rightarrow b)\right) \text { by Lemma 3.10 (1b). } \\
& =c \rightarrow\left(a^{\prime} \rightarrow\left(a^{\prime \prime} \rightarrow b\right)\right) \text { by }\left(\mathrm{I}_{2,0}\right) \\
& =c \rightarrow\left(a^{\prime} \rightarrow\left(b^{\prime} \rightarrow a^{\prime}\right)\right) \text { by Lemma } 3.1 \text { (b) } \\
& =c \rightarrow\left(b^{\prime} \rightarrow\left(a^{\prime} \rightarrow a^{\prime}\right)\right) \text { by Lemma } 3.1 \text { (回) } \\
& =c \rightarrow\left(\left(a^{\prime} \rightarrow a^{\prime}\right)^{\prime} \rightarrow b\right) \text { by Lemma 3.1 (b) } \\
& =c \rightarrow((a \rightarrow a) \rightarrow b) \text { by Lemma } 3.10 \text { (1b) } \\
& =((c \rightarrow a) \rightarrow a) \rightarrow b \quad \text { by }\left(C_{25}\right) \\
& =b^{\prime} \rightarrow((c \rightarrow a) \rightarrow a)^{\prime} \text { by Lemma } 3.1 \text { (ㄷ) } \\
& =b^{\prime} \rightarrow\left((c \rightarrow a)^{\prime} \rightarrow a^{\prime}\right) \text { by Lemma 3.10 (1b) } \\
& =b^{\prime} \rightarrow(a \rightarrow(c \rightarrow a)) \text { by Lemma } 3.1 \text { (ㄷ) } \\
& =b^{\prime} \rightarrow(c \rightarrow(a \rightarrow a)) \text { by Lemma } 3.1 \text { (回) } \\
& =b^{\prime} \rightarrow\left((a \rightarrow a)^{\prime} \rightarrow c^{\prime}\right) \text { by Lemma } 3.1 \text { (ㄷ) } \\
& =(a \rightarrow a)^{\prime} \rightarrow\left(b^{\prime} \rightarrow c^{\prime}\right) \text { by Lemma } 3.1 \text { (回) } \\
& =\left(b^{\prime} \rightarrow c^{\prime}\right)^{\prime} \rightarrow(a \rightarrow a) \quad \text { by Lemma } 3.1 \text { (b) } \\
& =(b \rightarrow c) \rightarrow(a \rightarrow a) \text { by Lemma 3.10 (1b) } \\
& =a \rightarrow((b \rightarrow c) \rightarrow a) \quad \text { by Lemma } 3.1 \text { (a) } \text {. }
\end{aligned}
$$

Thus,

$$
\mathcal{C}_{25} \subseteq \mathcal{D}_{25}
$$

Let $\mathbf{A} \in \mathcal{D}_{25}$ and $a, b, c \in A$. Then 


$$
\begin{aligned}
& ((a \rightarrow b) \rightarrow c) \rightarrow b=b^{\prime} \rightarrow((a \rightarrow b) \rightarrow c)^{\prime} \quad \text { by Lemma 3.1 (IC) } \\
& =b^{\prime} \rightarrow\left(c^{\prime} \rightarrow(a \rightarrow b)^{\prime}\right)^{\prime} \text { by Lemma } 3.1 \text { (IC) } \\
& =b^{\prime} \rightarrow\left(c^{\prime} \rightarrow\left(a^{\prime} \rightarrow b^{\prime}\right)\right)^{\prime} \text { by Lemma } 3.10 \text { (1b) } \\
& =b^{\prime} \rightarrow\left(c \rightarrow\left(a^{\prime} \rightarrow b^{\prime}\right)^{\prime}\right) \quad \text { by Lemma } 3.10 \text { (1b) } \\
& =b^{\prime} \rightarrow(c \rightarrow(a \rightarrow b)) \quad \text { by Lemma } 3.10 \text { (1b) } \\
& =b^{\prime} \rightarrow\left(c \rightarrow\left(a^{\prime \prime} \rightarrow b\right)\right) \text { by }\left(\overline{I_{2,0}}\right) \\
& =b^{\prime} \rightarrow\left(c \rightarrow\left(b^{\prime} \rightarrow a^{\prime}\right)\right) \text { by Lemma } 3.1(\mathrm{~b}) \\
& =b^{\prime} \rightarrow\left(b^{\prime} \rightarrow\left(c \rightarrow a^{\prime}\right)\right) \text { by Lemma } 3.1 \text { (国) } \\
& =b^{\prime} \rightarrow\left(c \rightarrow a^{\prime}\right) \quad \text { by Lemma } 2.4 \text { (国) } \\
& =b^{\prime} \rightarrow\left(a \rightarrow c^{\prime}\right) \quad \text { by Lemma } 3.1 \text { (d) } \\
& =a \rightarrow\left(b^{\prime} \rightarrow c^{\prime}\right) \quad \text { by Lemma } 3.1 \text { (国) } \\
& =a \rightarrow(b \rightarrow c)^{\prime} \quad \text { by Lemma } 3.10(1 \mathrm{~b}) \\
& =(b \rightarrow c) \rightarrow a^{\prime} \quad \text { by Lemma } 3.1 \text { (dd) } \\
& =(b \rightarrow(b \rightarrow c)) \rightarrow a^{\prime} \quad \text { by Lemma } 2.4 \text { (国) } \\
& =\left((b \rightarrow c)^{\prime} \rightarrow b^{\prime}\right) \rightarrow a^{\prime} \text { by Lemma 3.1 (ㄷ) } \\
& =((b \rightarrow c) \rightarrow b)^{\prime} \rightarrow a^{\prime} \quad \text { by Lemma } 3.10 \text { (1b) } \\
& =a \rightarrow((b \rightarrow c) \rightarrow b) \quad \text { by Lemma } 3.1 \text { (IC). }
\end{aligned}
$$

Consequently,

$$
\mathcal{D}_{25} \subseteq \mathcal{E}_{25} .
$$

Let $\mathbf{A} \in \mathcal{E}_{25}$ and $a, b, c \in A$. Then 


$$
\begin{aligned}
& (a \rightarrow a) \rightarrow(b \rightarrow c)=b \rightarrow((a \rightarrow a) \rightarrow c) \quad \text { by Lemma } 3.1 \text { (国) } \\
& =b \rightarrow\left(c^{\prime} \rightarrow(a \rightarrow a)^{\prime}\right) \quad \text { by Lemma } 3.1 \text { (ㄷ) } \\
& =b \rightarrow\left(c^{\prime} \rightarrow\left(a^{\prime} \rightarrow a^{\prime}\right)\right) \text { by Lemma 3.10 (1b) } \\
& =b \rightarrow\left(a^{\prime} \rightarrow\left(c^{\prime} \rightarrow a^{\prime}\right)\right) \quad \text { by Lemma } 3.1 \text { (国). } \\
& =b \rightarrow\left(\left(c^{\prime} \rightarrow a^{\prime}\right)^{\prime} \rightarrow a\right) \text { by Lemma } 3.1 \text { (b) } \\
& =b \rightarrow((c \rightarrow a) \rightarrow a) \text { by Lemma 3.10 (1b) } \\
& =((b \rightarrow c) \rightarrow a) \rightarrow a \quad \text { by }\left(E_{25}\right) \\
& =a^{\prime} \rightarrow((b \rightarrow c) \rightarrow a)^{\prime} \quad \text { by Lemma } 3.1 \text { (CC) } \\
& =a^{\prime} \rightarrow\left(a^{\prime} \rightarrow(b \rightarrow c)^{\prime}\right)^{\prime} \text { by Lemma } 3.1 \text { (ㄷ) } \\
& =a^{\prime} \rightarrow\left(a^{\prime} \rightarrow\left(b^{\prime} \rightarrow c^{\prime}\right)\right)^{\prime} \text { by Lemma } 3.10 \text { (10 } \\
& =a^{\prime} \rightarrow\left(a^{\prime} \rightarrow(c \rightarrow b)\right)^{\prime} \quad \text { by Lemma } 3.1 \text { (드 } \\
& =a^{\prime} \rightarrow\left(a \rightarrow(c \rightarrow b)^{\prime}\right) \text { by Lemma } 3.10 \text { (10) } \\
& =a \rightarrow\left(a^{\prime} \rightarrow(c \rightarrow b)^{\prime}\right) \quad \text { by Lemma } 3.1 \text { (国) } \\
& =a \rightarrow(a \rightarrow(c \rightarrow b))^{\prime} \quad \text { by Lemma 3.10 (1b) } \\
& =a \rightarrow(c \rightarrow(a \rightarrow b))^{\prime} \text { by Lemma } 3.1 \text { (国) } \\
& =a \rightarrow\left(c \rightarrow\left(a^{\prime} \rightarrow b^{\prime}\right)^{\prime}\right)^{\prime} \text { by Lemma 3.10 (10) } \\
& =a \rightarrow\left(\left(a^{\prime} \rightarrow b^{\prime}\right) \rightarrow c^{\prime}\right)^{\prime} \text { by Lemma } 3.1 \text { (dd) } \\
& =a \rightarrow\left((a \rightarrow b)^{\prime} \rightarrow c^{\prime}\right)^{\prime} \text { by Lemma 3.10 (1b) } \\
& =\left((a \rightarrow b)^{\prime} \rightarrow c^{\prime}\right) \rightarrow a^{\prime} \text { by Lemma 3.1 (d) } \\
& =((a \rightarrow b) \rightarrow c)^{\prime} \rightarrow a^{\prime} \text { by Lemma 3.10 (1b) } \\
& =a \rightarrow((a \rightarrow b) \rightarrow c) \text { by Lemma } 3.1 \text { (ㄷ) } \text {. }
\end{aligned}
$$

Therefore,

$$
\mathcal{E}_{25} \subseteq \mathcal{A}_{23}
$$

From (9), (10), (11), (12) and (13), we conclude that

$$
\mathcal{A}_{23}=\mathcal{A}_{25}=\mathcal{C}_{25}=\mathcal{D}_{25}=\mathcal{E}_{25} .
$$

(b) Using Lemma 3.1 (国) we can easily verify that $\mathcal{A}_{12}=\mathcal{B}_{13}$ and $\mathcal{F}_{13}=\mathcal{D}_{12}$.

Let $\mathbf{A} \in \mathcal{A}_{12}$ and $a, b, c \in A$. Then

$$
\begin{aligned}
a \rightarrow(b \rightarrow(c \rightarrow a)) & =b \rightarrow(a \rightarrow(c \rightarrow a)) \text { by Lemma 3.1 (国) } \\
& =b \rightarrow(c \rightarrow(a \rightarrow a)) \text { by Lemma 3.1 (国) } \\
& =b \rightarrow(c \rightarrow(0 \rightarrow a)) \text { by Lemma 3.11 (国) } \\
& =b \rightarrow(0 \rightarrow(c \rightarrow a)) \text { by Lemma 3.1 (国) } \\
& =0 \rightarrow(b \rightarrow(c \rightarrow a)) \text { by Lemma 3.1 (国) } \\
& =0 \rightarrow((b \rightarrow c) \rightarrow a) \text { by Lemma 3.11 (和) } \\
& =(b \rightarrow c) \rightarrow(0 \rightarrow a) \text { by Lemma 3.1 (国) } \\
& =(b \rightarrow c) \rightarrow(a \rightarrow a) \text { by Lemma 3.11 (国) } \\
& =a \rightarrow((b \rightarrow c) \rightarrow a) \text { by Lemma 3.1 (国). }
\end{aligned}
$$


Therefore,

$$
\mathcal{A}_{12} \subseteq \mathcal{D}_{12} .
$$

Let $\mathbf{A} \in \mathcal{D}_{12}$ and $a, b, c \in A$. Then

$$
\begin{aligned}
& ((a \rightarrow b) \rightarrow c) \rightarrow a \\
& =\left(\left(a^{\prime} \rightarrow(a \rightarrow b)\right) \rightarrow(c \rightarrow a)^{\prime}\right)^{\prime} \quad \text { by (I) } \\
& =\left(\left(a \rightarrow\left(a^{\prime} \rightarrow b\right)\right) \rightarrow(c \rightarrow a)^{\prime}\right)^{\prime} \quad \text { by Lemma } 3.1 \text { (a) } \\
& =\left(\left(a \rightarrow\left(b^{\prime} \rightarrow a\right)\right) \rightarrow(c \rightarrow a)^{\prime}\right)^{\prime} \quad \text { by Lemma } 3.1 \text { (b) } \\
& =\left(\left(b^{\prime} \rightarrow(a \rightarrow a)\right) \rightarrow(c \rightarrow a)^{\prime}\right)^{\prime} \quad \text { by Lemma } 3.1 \text { (国) } \\
& =\left(\left(b^{\prime} \rightarrow(0 \rightarrow a)\right) \rightarrow(c \rightarrow a)^{\prime}\right)^{\prime} \text { by Lemma 3.11 (a) } \\
& =\left(\left(0 \rightarrow\left(b^{\prime} \rightarrow a\right)\right) \rightarrow(c \rightarrow a)^{\prime}\right)^{\prime} \quad \text { by Lemma } 3.1 \text { (国) } \\
& =\left(\left(0 \rightarrow\left(a^{\prime} \rightarrow b\right)\right) \rightarrow(c \rightarrow a)^{\prime}\right)^{\prime} \quad \text { by Lemma } 3.1 \text { (b) } \\
& =\left(\left(0 \rightarrow\left(0 \rightarrow\left(a^{\prime} \rightarrow b\right)\right)\right) \rightarrow(c \rightarrow a)^{\prime}\right)^{\prime} \quad \text { by Lemma } 2.4 \text { (EE) } \\
& =\left(\left((c \rightarrow a)^{\prime} \rightarrow\left(0 \rightarrow\left(a^{\prime} \rightarrow b\right)\right)\right) \rightarrow(c \rightarrow a)^{\prime}\right)^{\prime} \text { by Lemma } 2.4 \text { (IC) } \\
& =\left(\left(0 \rightarrow\left((c \rightarrow a)^{\prime} \rightarrow\left(a^{\prime} \rightarrow b\right)\right)\right) \rightarrow(c \rightarrow a)^{\prime}\right)^{\prime} \text { by Lemma } 3.1 \text { (国) }
\end{aligned}
$$


and

$$
\begin{aligned}
& \left(\left(0 \rightarrow\left((c \rightarrow a)^{\prime} \rightarrow\left(a^{\prime} \rightarrow b\right)\right)\right) \rightarrow(c \rightarrow a)^{\prime}\right)^{\prime} \\
& =\left(\left(\left(0 \rightarrow(c \rightarrow a)^{\prime}\right) \rightarrow\left(0 \rightarrow\left(a^{\prime} \rightarrow b\right)\right)\right) \rightarrow(c \rightarrow a)^{\prime}\right)^{\prime} \\
& \text { by Lemma } 2.4 \text { (hㅡ) and (d) } \\
& =\left(\left((0 \rightarrow(c \rightarrow a)) \rightarrow\left(0 \rightarrow\left(a^{\prime} \rightarrow b\right)\right)\right) \rightarrow(c \rightarrow a)^{\prime}\right)^{\prime} \quad \text { by Lemma } 3.11 \text { (国) } \\
& =\left(\left(0 \rightarrow\left((c \rightarrow a) \rightarrow\left(a^{\prime} \rightarrow b\right)\right)\right) \rightarrow(c \rightarrow a)^{\prime}\right)^{\prime} \\
& \text { by Lemma } 2.4 \text { (h) and (did) } \\
& =\left(\left(0 \rightarrow\left(\left((c \rightarrow a) \rightarrow a^{\prime}\right) \rightarrow b\right)\right) \rightarrow(c \rightarrow a)^{\prime}\right)^{\prime} \\
& =\left(\left(\left(0 \rightarrow\left((c \rightarrow a) \rightarrow a^{\prime}\right)\right) \rightarrow(0 \rightarrow b)\right) \rightarrow(c \rightarrow a)^{\prime}\right)^{\prime} \\
& \text { by Lemma } 2.4 \text { (h) and (d) } \\
& =\left(\left(\left(0 \rightarrow\left(a^{\prime} \rightarrow(c \rightarrow a)\right)\right) \rightarrow(0 \rightarrow b)\right) \rightarrow(c \rightarrow a)^{\prime}\right)^{\prime} \quad \text { by Lemma } 3.11 \text { (b) } \\
& =\left(((0 \rightarrow(c \rightarrow a)) \rightarrow(0 \rightarrow b)) \rightarrow(c \rightarrow a)^{\prime}\right)^{\prime} \quad \text { by Lemma 2.4 (j]) } \\
& =\left(\left(\left(0 \rightarrow(c \rightarrow a)^{\prime}\right) \rightarrow(0 \rightarrow b)\right) \rightarrow(c \rightarrow a)^{\prime}\right)^{\prime} \quad \text { by Lemma } 3.11 \text { (国) } \\
& =\left(\left(0 \rightarrow\left((c \rightarrow a)^{\prime} \rightarrow b\right)\right) \rightarrow(c \rightarrow a)^{\prime}\right)^{\prime} \\
& \text { by Lemma } 2.4 \text { (hin) and (d) } \\
& =\left(\left((c \rightarrow a)^{\prime} \rightarrow(0 \rightarrow b)\right) \rightarrow(c \rightarrow a)^{\prime}\right)^{\prime} \\
& =\left((0 \rightarrow(0 \rightarrow b)) \rightarrow(c \rightarrow a)^{\prime}\right)^{\prime} \\
& =\left(\left(0 \rightarrow(0 \rightarrow b)^{\prime}\right) \rightarrow(c \rightarrow a)^{\prime}\right)^{\prime} \\
& =\left(\left((0 \rightarrow b) \rightarrow 0^{\prime}\right) \rightarrow(c \rightarrow a)^{\prime}\right)^{\prime} \\
& =\left(\left((0 \rightarrow b) \rightarrow(c \rightarrow a)^{\prime \prime}\right) \rightarrow(c \rightarrow a)^{\prime}\right)^{\prime} \\
& =\left(((0 \rightarrow b) \rightarrow(c \rightarrow a)) \rightarrow(c \rightarrow a)^{\prime}\right)^{\prime} \\
& =\left(\left((c \rightarrow a)^{\prime} \rightarrow(0 \rightarrow b)^{\prime}\right) \rightarrow(c \rightarrow a)^{\prime}\right)^{\prime} \\
& =\left(\left(((c \rightarrow a) \rightarrow 0) \rightarrow(0 \rightarrow b)^{\prime}\right) \rightarrow(c \rightarrow a)^{\prime}\right)^{\prime} \\
& =\left(\left(((c \rightarrow a) \rightarrow 0) \rightarrow(0 \rightarrow b)^{\prime}\right) \rightarrow\left(0^{\prime} \rightarrow(c \rightarrow a)\right)^{\prime}\right)^{\prime} \text { by Lemma } 2.2 \text { (国) } \\
& =\left((0 \rightarrow b)^{\prime} \rightarrow 0^{\prime}\right) \rightarrow(c \rightarrow a) \\
& =(0 \rightarrow(0 \rightarrow b)) \rightarrow(c \rightarrow a) \\
& =(0 \rightarrow b) \rightarrow(c \rightarrow a) \\
& =c \rightarrow((0 \rightarrow b) \rightarrow a) \\
& =c \rightarrow((a \rightarrow b) \rightarrow a) \\
& =(a \rightarrow b) \rightarrow(c \rightarrow a)
\end{aligned}
$$

Hence

$$
\mathcal{D}_{12} \subseteq \mathcal{D}_{35}
$$

Next, let $\mathbf{A} \in \mathcal{D}_{35}$ and $a, b, c \in A$. Then

$a \rightarrow((a \rightarrow b) \rightarrow c)$ 


$$
\begin{aligned}
& =(a \rightarrow b) \rightarrow(a \rightarrow c) \\
& =\left[\left((a \rightarrow c)^{\prime} \rightarrow a\right) \rightarrow(b \rightarrow(a \rightarrow c))^{\prime}\right)^{\prime} \\
& =\left((((a \rightarrow c) \rightarrow 0) \rightarrow a) \rightarrow(b \rightarrow(a \rightarrow c))^{\prime}\right)^{\prime} \\
& =\left(((a \rightarrow c) \rightarrow(0 \rightarrow a)) \rightarrow(b \rightarrow(a \rightarrow c))^{\prime}\right)^{\prime} \text { by }\left(D_{35}\right) \\
& =\left((0 \rightarrow((a \rightarrow c) \rightarrow a)) \rightarrow(b \rightarrow(a \rightarrow c))^{\prime}\right)^{\prime} \text { by Lemma } 3.1 \text { (国) } \\
& =\left((0 \rightarrow((0 \rightarrow c) \rightarrow a)) \rightarrow(b \rightarrow(a \rightarrow c))^{\prime}\right)^{\prime} \text { by Lemma } 2.4 \text { (IC) } \\
& =\left(((0 \rightarrow c) \rightarrow(0 \rightarrow a)) \rightarrow(b \rightarrow(a \rightarrow c))^{\prime}\right)^{\prime} \text { by Lemma } 3.1 \text { (a) } \\
& =\left((0 \rightarrow(c \rightarrow a)) \rightarrow(b \rightarrow(a \rightarrow c))^{\prime}\right)^{\prime} \\
& \text { by Lemma } 2.4 \text { (h) and (dd) } \\
& =\left((0 \rightarrow(a \rightarrow c)) \rightarrow(b \rightarrow(a \rightarrow c))^{\prime}\right)^{\prime} \quad \text { by Lemma 3.11 (b) } \\
& =\left(\left((a \rightarrow c)^{\prime} \rightarrow 0^{\prime}\right) \rightarrow(b \rightarrow(a \rightarrow c))^{\prime}\right)^{\prime} \quad \text { by Lemma } 2.3 \text { (b) } \\
& =\left(0^{\prime} \rightarrow b\right) \rightarrow(a \rightarrow c) \\
& =b \rightarrow(a \rightarrow c) \\
& =a \rightarrow(b \rightarrow c) \\
& =a \rightarrow(a \rightarrow(b \rightarrow c)) \\
& \text { using (I) } \\
& \text { by Lemma } 2.2 \text { (国) } \\
& \text { by Lemma } 3.1 \text { (国) } \\
& \text { by Lemma } 2.4 \text { (回). }
\end{aligned}
$$

As a consequence, we have that

$$
\mathcal{D}_{35} \subseteq \mathcal{A}_{12} .
$$

Therefore, using (14), (15) and (16) we get that

$$
\mathcal{A}_{12}=\mathcal{D}_{12}=\mathcal{D}_{35}
$$

(c) Let $\mathbf{A} \in \mathcal{A}_{35}$ and $a, b, c \in A$. Then

$$
\begin{aligned}
& ((a \rightarrow b) \rightarrow c) \rightarrow c=\left(\left(c^{\prime} \rightarrow(a \rightarrow b)\right) \rightarrow(c \rightarrow c)^{\prime}\right)^{\prime} \quad \text { by }(\mathrm{I}) \\
& =\left((c \rightarrow c) \rightarrow\left(c^{\prime} \rightarrow(a \rightarrow b)\right)^{\prime}\right)^{\prime} \text { by Lemma 3.1 (dd) } \\
& =\left((c \rightarrow c) \rightarrow\left(c^{\prime} \rightarrow(a \rightarrow b)\right)\right)^{\prime \prime} \quad \text { by }\left(A_{35}\right) \\
& =(c \rightarrow c) \rightarrow\left(c^{\prime} \rightarrow(a \rightarrow b)\right) \quad \text { by }\left(\mathrm{I}_{2,0}\right) \\
& =(c \rightarrow c) \rightarrow\left(a \rightarrow\left(c^{\prime} \rightarrow b\right)\right) \quad \text { by Lemma } 3.1 \text { (国) } \\
& =a \rightarrow\left((c \rightarrow c) \rightarrow\left(c^{\prime} \rightarrow b\right)\right) \quad \text { by Lemma 3.1 (回) } \\
& =a \rightarrow\left((c \rightarrow c) \rightarrow\left(c^{\prime} \rightarrow b\right)^{\prime \prime}\right) \quad \text { by }\left(\mathrm{I}_{2,0}\right) \\
& =a \rightarrow\left((c \rightarrow c) \rightarrow\left(c^{\prime} \rightarrow b\right)^{\prime}\right)^{\prime} \quad \text { by }\left(A_{35}\right) \\
& =a \rightarrow\left(\left(c^{\prime} \rightarrow b\right) \rightarrow(c \rightarrow c)^{\prime}\right)^{\prime} \quad \text { by Lemma } 3.1 \text { (d) } \\
& =a \rightarrow((b \rightarrow c) \rightarrow c) \quad \text { by (I). }
\end{aligned}
$$

Then

$$
\mathcal{A}_{35} \subseteq \mathcal{F}_{25}
$$


For the converse, suppose $\mathbf{A} \in \mathcal{F}_{25}$ and $a, b, c \in A$. Then we have that

$$
\begin{array}{rlr}
0 \rightarrow(a \rightarrow a) & =0 \rightarrow\left(\left(0^{\prime} \rightarrow a\right) \rightarrow a\right) & \text { by Lemma } 2.2 \text { (国) } \\
& =\left(\left(0 \rightarrow 0^{\prime}\right) \rightarrow a\right) \rightarrow a & \text { by }\left(F_{25}\right) \\
& =\left(\left(0^{\prime \prime} \rightarrow 0^{\prime}\right) \rightarrow a\right) \rightarrow a & \text { by }\left(\bar{I}_{2,0}\right) \\
& =a \rightarrow a & \text { by Lemma } 2.2(\text { dd }) .
\end{array}
$$

Hence,

$$
\mathbf{A} \models 0 \rightarrow(x \rightarrow x) \approx x \rightarrow x .
$$

Using Lemma 3.4 we have that $\mathbf{A} \in \mathcal{A}_{35}$. Thus,

$$
\mathcal{F}_{25} \subseteq \mathcal{A}_{35}
$$

(d) Let $\mathbf{A} \in \mathcal{S}$ and $a, b, c \in A$. Then

$$
\begin{aligned}
& a \rightarrow((b \rightarrow a) \rightarrow c)=(b \rightarrow a) \rightarrow(a \rightarrow c) \quad \text { by Lemma } 3.1 \text { (国) } \\
& =\left(\left((a \rightarrow c)^{\prime} \rightarrow b\right) \rightarrow(a \rightarrow(a \rightarrow c))^{\prime}\right)^{\prime} \quad \text { by }(\mathrm{I}) \\
& =\left(\left((a \rightarrow c)^{\prime} \rightarrow b\right) \rightarrow(a \rightarrow c)^{\prime}\right)^{\prime} \quad \text { by Lemma } 2.4 \text { (国) } \\
& =\left(\left(b^{\prime} \rightarrow(a \rightarrow c)\right) \rightarrow(a \rightarrow c)^{\prime}\right)^{\prime} \quad \text { by Lemma } 3.1(\mathrm{~b}) \\
& =\left(\left(b^{\prime} \rightarrow\left(a^{\prime \prime} \rightarrow c\right)\right) \rightarrow(a \rightarrow c)^{\prime}\right)^{\prime} \quad \text { by }\left(\mathrm{I}_{2,0}\right) \\
& =\left(\left(b^{\prime} \rightarrow\left(c^{\prime} \rightarrow a^{\prime}\right)\right) \rightarrow(a \rightarrow c)^{\prime}\right)^{\prime} \quad \text { by Lemma } 3.1 \text { (b) } \\
& =\left(\left(c^{\prime} \rightarrow\left(b^{\prime} \rightarrow a^{\prime}\right)\right) \rightarrow(a \rightarrow c)^{\prime}\right)^{\prime} \quad \text { by Lemma } 3.1 \text { (国) } \\
& =\left(\left(c^{\prime} \rightarrow(a \rightarrow b)\right) \rightarrow(a \rightarrow c)^{\prime}\right)^{\prime} \quad \text { by Lemma } 3.1 \text { (IC) } \\
& =((a \rightarrow b) \rightarrow a) \rightarrow c \quad \text { by (I). }
\end{aligned}
$$

Thus, $\mathcal{S} \subseteq \mathcal{B}_{25}$, implying that $\mathcal{B}_{25}=\mathcal{S}$. This completes the proof.

Since $\mathcal{S} \mathcal{L}$ coincides with some of Bol-Moufang varieties and $\mathcal{S}$ coincides with $\mathcal{B}_{25}$, we regard both of them as varieties of Bol-Moufang type, relative to $\mathcal{S}$.

We are now ready to present our main result of this paper.

THEOREM 4.2. There are 5 nontrivial varieties of Bol-Moufang type, relative to $\mathcal{S}$, that are distinct from each other: $\mathcal{S} \mathcal{L}, \mathcal{A}_{12}, \mathcal{A}_{23}, \mathcal{F}_{25}$ and $\mathcal{S}$. These varieties satisfy the following inclusions:

(a) $\mathcal{S} \mathcal{L} \subset \mathcal{A}_{23} \subset \mathcal{F}_{25}$

(b) $\mathcal{S L} \subset \mathcal{A}_{12}$

(c) $\mathcal{B A} \subset \mathcal{A}_{12} \subset \mathcal{F}_{25}$, 
(d) $\mathcal{F}_{25} \subset \mathcal{I}_{2,0} \cap \mathcal{M C}$,

(e) $\mathcal{S L}=\mathcal{A}_{23} \cap \mathcal{A}_{12}$.

Proof. (a) By Lemma 3.6 we know that $\mathcal{S} \mathcal{L} \subseteq \mathcal{A}_{23}$.

The following example shows that the inclusion is proper:

\begin{tabular}{r|cccc}
$\rightarrow:$ & 0 & 1 & 2 & 3 \\
\hline 0 & 0 & 1 & 2 & 3 \\
1 & 2 & 3 & 2 & 3 \\
2 & 1 & 1 & 3 & 3 \\
3 & 3 & 3 & 3 & 3
\end{tabular}

Next, we wish to show that $\mathcal{A}_{23} \subseteq \mathcal{F}_{25}$. So, let $\mathbf{A} \in \mathcal{A}_{23}$ and $a \in A$.

Then

$$
\begin{aligned}
& 0 \rightarrow(a \rightarrow a)=(0 \rightarrow a) \rightarrow(0 \rightarrow a) \text { by Lemma } 2.4 \text { (h) and (dd) } \\
& =0 \rightarrow((0 \rightarrow a) \rightarrow a) \text { by Lemma } 3.1 \text { (a) } \\
& =(0 \rightarrow 0) \rightarrow(a \rightarrow a) \text { by }\left(A_{23}\right) \\
& =a \rightarrow a \quad \text { by Lemma } 2.2 \text { (国). }
\end{aligned}
$$

By Lemma 3.4 and Theorem 4.1 (IC), we have $\mathcal{A}_{23} \subseteq \mathcal{F}_{25}$.

The following example shows that the inclusion is proper:

\begin{tabular}{r|rr}
$\rightarrow:$ & 0 & 1 \\
\hline 0 & 1 & 1 \\
1 & 0 & 1
\end{tabular}

(b) By Lemma 3.6 we know that $\mathcal{S L} \subseteq \mathcal{A}_{12}$.

The following example shows that the inclusion is proper:

\begin{tabular}{r|rr}
$\rightarrow:$ & 0 & 1 \\
\hline 0 & 1 & 1 \\
1 & 0 & 1
\end{tabular}

(c) By Lemma 3.5 we know that $\mathcal{B A} \subseteq \mathcal{A}_{12}$.

The following example shows that the inclusion is proper:

\begin{tabular}{r|rr}
$\rightarrow:$ & 0 & 1 \\
\hline 0 & 0 & 1 \\
1 & 1 & 1
\end{tabular}


To prove $\mathcal{A}_{12} \subseteq \mathcal{F}_{25}$, we let $\mathbf{A} \in \mathcal{A}_{12}$ and $a \in A$. Then

$$
\begin{aligned}
0 \rightarrow(a \rightarrow a) & =(0 \rightarrow a) \rightarrow(0 \rightarrow a) & & \text { by Lemma } 2.4 \text { (h) and (d) } \\
& =0 \rightarrow(0 \rightarrow a) & & \text { by Lemma } 3.11 \text { (国) } \\
& =0 \rightarrow a & & \text { by Lemma } 2.4 \text { (国) } \\
& =a \rightarrow a & & \text { by Lemma } 3.11 \text { (国). }
\end{aligned}
$$

By Lemma 3.4 and Theorem 4.1 (ㄷ), $\mathcal{A}_{12} \subseteq \mathcal{F}_{25}$.

The following example shows that the inclusion is proper:

\begin{tabular}{r|cccc}
$\rightarrow:$ & 0 & 1 & 2 & 3 \\
\hline 0 & 0 & 1 & 2 & 3 \\
1 & 2 & 3 & 2 & 3 \\
2 & 1 & 1 & 3 & 3 \\
3 & 3 & 3 & 3 & 3
\end{tabular}

(d) The following example shows that the inclusion is proper:

\begin{tabular}{r|rrr}
$\rightarrow:$ & 0 & 1 & 2 \\
\hline 0 & 2 & 2 & 2 \\
1 & 1 & 1 & 2 \\
2 & 0 & 1 & 2
\end{tabular}

(e) Let $\mathrm{A} \in \mathcal{A}_{23} \cap \mathcal{A}_{12}$ and $a \in A$. Then

$$
\begin{aligned}
a & =a^{\prime \prime} & & \text { by }\left[\mathrm{I}_{2,0}\right) \\
& =a^{\prime} \rightarrow 0 & & \\
& =0^{\prime} \rightarrow\left(a^{\prime} \rightarrow 0\right) & & \text { by Lemma } 2.2 \text { (回) } \\
& =(0 \rightarrow 0) \rightarrow\left(a^{\prime} \rightarrow 0\right) & & \\
& =0 \rightarrow\left(\left(0 \rightarrow a^{\prime}\right) \rightarrow 0\right) & & \text { by }\left(A_{23}\right) \\
& =0 \rightarrow\left(0 \rightarrow\left(a^{\prime} \rightarrow 0\right)\right) & & \text { by }\left(A_{12}\right) \\
& =0 \rightarrow\left(a^{\prime} \rightarrow 0\right) & & \text { by Lemma } 2.4(\text { (远) } \\
& =0 \rightarrow a^{\prime \prime} & & \\
& =0 \rightarrow a . & & \text { by }\left(\mathrm{I}_{2,0}\right)
\end{aligned}
$$

Hence

$$
\mathbf{A} \models x \approx 0 \rightarrow x
$$

Consequently,

$$
\mathbf{A} \models 0 \approx 0^{\prime}
$$


Therefore,

$$
\begin{aligned}
& a \rightarrow a=(a \rightarrow a)^{\prime \prime} \quad \text { by }\left(\mathrm{I}_{2,0}\right) \\
& =((a \rightarrow a) \rightarrow 0)^{\prime} \\
& =\left((a \rightarrow a) \rightarrow 0^{\prime}\right)^{\prime} \quad \text { by (18) } \\
& =((a \rightarrow a) \rightarrow(0 \rightarrow 0))^{\prime} \\
& =(a \rightarrow((a \rightarrow 0) \rightarrow 0))^{\prime} \text { by }\left(A_{23}\right) \\
& =(a \rightarrow(a \rightarrow(0 \rightarrow 0)))^{\prime} \text { by }\left(A_{12}\right) \\
& =(a \rightarrow(0 \rightarrow 0))^{\prime} \quad \text { by Lemma } 2.4 \text { (回) } \\
& =\left(a \rightarrow 0^{\prime}\right)^{\prime} \\
& =\left(0 \rightarrow a^{\prime}\right)^{\prime} \quad \text { by Lemma } 2.3(\underline{b}) \\
& =\left(a^{\prime}\right)^{\prime} \quad \text { by (17) } \\
& =a . \quad \text { by }\left(\mathrm{I}_{2,0}\right.
\end{aligned}
$$

Thus, $\mathbf{A} \models x \rightarrow x \approx x$. Hence, from Lemma 3.2 and Lemma 2.1. $\mathbf{A} \in \mathcal{S} \mathcal{L}$. In view of Lemma 3.6 the proof is complete.

From the preceding theorem, it is easy to see that the poset (in fact, $\wedge$-semilattice) of varieties of Bol-Moufang type, together with the variety $\mathcal{B A}$ of Boolean algebras, under inclusion is as shown below:

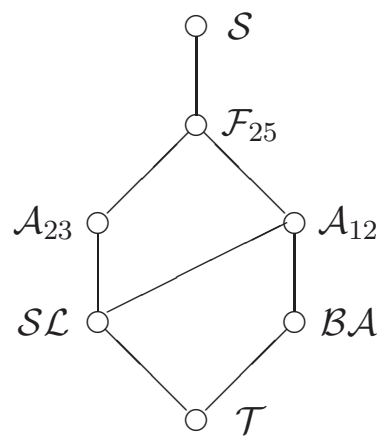

It is worthwhile to point out that our investigations into Bol-Moufang identities relative to $\mathcal{S}$, have revealed three new varieties, namely $\mathcal{A}_{12}, \mathcal{A}_{23}$ and $\mathcal{F}_{25}$, hitherto unknown.

In CS16d], we investigate all the remaining weak associative laws of size $\leq 4$ which will complement the results of this paper.

We conclude with the remark that it would be of interest to investigate the weak associative identities, and in particular, the identities of BolMoufang type, relative to $\mathcal{I}$ and other (important) subvarieties of $\mathcal{I}$ (see the 
Problem mentioned in the introduction).

Acknowledgment: The first author wants to thank the institutional support of CONICET (Consejo Nacional de Investigaciones Científicas y Técnicas). The authors wish to express their indebtedness to the anonymous referees for their careful reading of the paper.

\section{Compliance with Ethical Standards:}

Conflict of Interest: The first author declares that he has no conflict of interest. The second author declares that he has no conflict of interest.

Ethical approval: This article does not contain any studies with human participants or animals performed by any of the authors.

Funding: The work of Juan M. Cornejo was supported by CONICET (Consejo Nacional de Investigaciones Cientificas y Tecnicas) and Universidad Nacional del Sur. Hanamantagouda P. Sankappanavar did not receive any specific grant from funding agencies in the public, commercial, or not-for-profit sectors.

\section{References}

[BD74] Balbes R, Dwinger, PH (1974) Distributive lattices. Univ. of Missouri Press, Columbia.

[Be34] Bernstein BA (1934) A set of four postulates for Boolean algebras in terms of the implicative operation. Trans. Amer. Math. Soc. 36, 876884 .

[BS81] Burris S, Sankappanavar HP (1981) A course in universal Algebra. Springer-Verlag, New York. The free, corrected version (2012) is available online as a PDF file at math.uwaterloo.ca/ snburris.

[CS16] Cornejo JM, Sankappanavar HP (2017) On implicator groupoids. Algebra univers. 77(2), 125-146.

doi:10.1007/s00012-017-0429-0. arXiv:1509.03774. 
[CS16a] Cornejo JM, Sankappanavar HP (2016) Order in implication zroupoids. Stud Logica. 104, 417-453. doi: 10.1007/s11225-015-96468.

[CS16b] Cornejo JM, Sankappanavar HP (2016) Semisimple varieties of implication zroupoids. Soft Comput. 20, 3139-3151. doi: 10.1007/s00500015-1950-8.

[CS16c] Cornejo JM, Sankappanavar HP (2016) On derived algebras and subvarieties of implication zroupoids. Soft Comput. doi: 10.1007/s00500-016-2421-6, pages 1-20.

[CS16d] Cornejo JM, Sankappanavar HP (2017) Symmetric implication zroupoids and the weak associative laws (Submitted).

[Fe69] Fenyves F (1969) Extra loops. II. Publ. Math. Debrecen 16, 187-192.

[Ku96] Kunen K (1996) Quasigroups, loops, and associative laws. J. Algebra 185, 194-204. doi: 10.1006/jabr.1996.0321.

[Mc] McCune W (2005-2010) Prover9 and Mace4. URL: http://www.cs.unm.edu/mccune/prover9/

[PV05a] Phillips JD, Vojtechovsky P (2005) The varieties of loops of BolMoufang type, Algebra univers. 54, 259-271. doi: 10.1007/s00012-0051941-1.

[PV05b] Phillips JD, Vojtechovsky P (2005) The varieties of quasigroups of Bol-Moufang type: an equational reasoning approach. J. Algebra. 293, 17-33 (2005).

[R74] Rasiowa H (1974) An algebraic approach to non-classical logics. North-Holland, Amsterdam.

[San12] Sankappanavar HP (2012) De Morgan algebras: New perspectives and applications. Sci. Math. Jpn. 75(1): 21-50.

JuAn M. CORnejo

Departamento de Matemática

Universidad Nacional del Sur

Alem 1253, Bahía Blanca, Argentina

INMABB - CONICET 
jmcornejo@uns.edu.ar

Hanamantagouda P. SANKappanavaR

Department of Mathematics

State University of New York

New Paltz, New York 12561

U.S.A.

sankapph@newpaltz.edu 